\title{
Impacts of stellar evolution and dynamics on the habitable zone: The role of rotation and magnetic activity
}

\author{
F. Gallet ${ }^{1}$, C. Charbonnel ${ }^{1,2}$, L. Amard ${ }^{1,4}$, S. Brun ${ }^{3}$, A. Palacios ${ }^{4}$, and S. Mathis ${ }^{3}$ \\ 1 Department of Astronomy, University of Geneva, Chemin des Maillettes 51, 1290 Versoix, Switzerland \\ e-mail: Florian.gallet@unige.ch \\ 2 IRAP, UMR 5277, CNRS and Université de Toulouse, 14 Av. E. Belin, 31400 Toulouse, France \\ 3 Laboratoire AIM Paris-Saclay, CEA/DRF-Université Paris Diderot-CNRS, IRFU/SAp Centre de Saclay, 91191 Gif-sur-Yvette, \\ France \\ 4 LUPM, UMR 5299, Université Montpellier/CNRS, 34095 Montpellier, France
}

Received 1 June 2016 / Accepted 3 August 2016

\begin{abstract}
Context. With the ever growing number of detected and confirmed exoplanets, the probability of finding a planet that looks like the Earth increases continuously. While it is clear that the presence of a planet in the habitable zone does not imply the planet is habitable, a systematic study of the evolution of the habitable zone is required to account for its dependence on stellar parameters.

Aims. In this article, we aim to provide the community with the dependence of the habitable zone upon the stellar mass, metallicity, rotation, and for various prescriptions of the limits of the habitable zone.

Methods. We use stellar evolution models computed with the code STAREVOL, which includes the most current physical mechanisms of internal transport of angular momentum and external wind braking, to study the evolution of the habitable zone and the continuously habitable zone limits.

Results. The stellar parameters mass and metallicity affect the habitable zone limits most dramatically. Conversely, for a given stellar mass and metallicity, stellar rotation has only a marginal effect on these limits and does not modify the width of the habitable zone. Moreover, and as expected in the main-sequence phase and for a given stellar mass and metallicity, the habitable zone limits remain almost constant, and this confirms the usual assumptions of a relative constancy of these limits during that phase. The evolution of the habitable zone limits is also correlated to the evolution of the stellar activity (through the Rossby number), which depends on the stellar mass considered. While the magnetic activity has negligible consequence in the case of more massive stars, these effects may have a strong impact on the habitability of a planet around M-dwarf stars. Thus, stellar activity cannot be neglected and may have a strong impact on the development of life during the early stage of the continuously habitable zone phase of low-mass stars. Using observed trends of stellar magnetic field strength, we also constrain the planetary magnetic field (at the zero order) required for a sufficient magnetospheric protection during the whole stellar evolution.

Conclusions. We explain for the first time the systematic dependence of planet habitability on stellar parameters along the full evolution of low- and intermediate-mass stars. These results can be used as physical inputs for a first order estimation of exoplanetary habitability.
\end{abstract}

Key words. planet-star interactions - stars: activity - stars: solar-type - stars: low-mass - planets and satellites: physical evolution stars: evolution

\section{Introduction}

With more than 3000 exoplanets detected inside a broad range of configurations in terms of distance from the star, size, mass, and atmospheric conditions, the probability of finding more habitable planets may dramatically increase in near future.

As a result of the improving accuracy of modern observational techniques, such as the radial velocity and transit methods, newly detected planets have continuously reached smaller radii and masses since the first confirmed exoplanet $51 \mathrm{Peg} \mathrm{b}$ $\left(\approx 150 M_{\oplus}\right)$ discovered by Mayor \& Queloz (1995). At the beginning, observed exoplanets were only found close to their host star (later named hot Jupiters), but now the detection of telluric planets is starting to be a frequent occurence ${ }^{1}$ for example, Gliese 581 c (Udry et al. 2007), Gliese 581 e (Mayor et al. 2009), Kepler-9 d (Holman et al. 2010), Kepler-10 b

\footnotetext{
1 http://exoplanets.org/
}

(Batalha et al. 2011), Kepler-11 f (Lissauer et al. 2011), Kepler$37 \mathrm{~d}$ (Borucki et al. 2011), alpha Cen B b (Dumusque et al. 2012), Kepler-186 f (Quintana et al. 2014), and Kepler-438 b (Torres et al. 2015).

Among these observed objects, there is Kepler-438 b (Torres et al. 2015), which was recently confirmed to be inside the habitable zone of its host star Kepler-438, an M-dwarf star with a mass of $0.544 M_{\odot}$ and a radius of $0.520 R_{\odot}$. The radius of Kepler-438 b is very close to the Earth's radius (1.12 $\left.R_{\oplus}\right)$, and the probability that it possesses a rocky internal structure is estimated to be $69.6 \%$ (Torres et al. 2015). Such peculiar star-planet system is very interesting to analyse since in that configuration, i.e. where the planet is relatively close to its star (around $0.16 \mathrm{AU}$ for Kepler-438 b), both magnetic and tidal interactions between the two bodies are not negligible (e.g. Strugarek et al. 2014, 2015; Mathis 2015; Bolmont \& Mathis 2016) and could imply tidal dissipation, spin-orbit resonance, and synchronisation 
of the planet rotation period (Heller et al. 2011; Quintana et al. 2014).

Studying and constraining habitability of these kind of starplanet systems is thus difficult, but interesting as every physical process that play even a minor role in the close environment of the star must be included.

Life can be found in an extremely wide variety of conditions. Even on Earth, life forms can be found from extremophile up to mesophile organisms. Since "life" is a process rather than a substance, providing a definition for it is not straightforward. However, the development of life requires three key ingredients: a source of energy, some organic material, and a solvent (Forget 2013). Life form beyond the Earth boundary is completely unknown (yet) and naturally leads to habitable zone conditions described for the kinds of organisms found on Earth. Despite this geocentric definition, however, the habitable zone is generally defined in astrophysics as the orbital area around a star where liquid water can be present at the surface of the planet (Hart 1976; Kasting et al. 1993; Leconte et al. 2015; Gallet et al. 2016). Indeed, water is one of the main ingredients for organisms to metabolise and reproduce (Kasting 2010; Güdel et al. 2014).

A planet is called habitable when its orbital radius lies inside the habitable zone and the conditions of habitability are fulfilled. These conditions are not yet defined well and are linked to complexification processes. For instance, the presence of a planetary magnetosphere is thought to have a strong impact in helping complexification through magnetic shielding (Vidotto et al. 2013, 2015; Strugarek et al. 2014, 2015). While the temporal evolution of the habitable zone is often not taken into account in the study of planetary habitability (Kasting et al. 1993; Leconte et al. 2013b), the earlier and subsequent evolution of the habitable zone limits closely follow the inherent evolution of the host-star parameters (Gallet et al. 2016). This evolution can be very fast during peculiar phases of the stellar life, so that a planet seen in the habitable zone at a time $t$ can leave it in a few Myr. This leads to another important concept, which is the continuously habitable zone limits; such limits are linked to the time required for a planet inside the habitable zone to favour the emergence of complex organisms (Kasting et al. 1993). The minimum time required by "life" for complexification is not well known and is usually fixed, assuming Earth-like life (at a value between 2 and 4 Gyr; see Valle et al. 2014; Truitt et al. 2015).

Because of these complex habitability conditions, a planet found inside the habitable zone is not necessarily considered habitable. However, the prime condition to analyse when studying the habitability of a recently detected planet is whether or not this planet is inside the habitable zone. We therefore need to perfectly comprehend the evolution of the planet as a function of the variation of stellar parameters.

The aim of this article is to study the impact of stellar parameters such as metallicity, mass, and rotation all along the evolution of low- and intermediate mass stars on the limits of the habitable zone (hereafter HZ, see also Gallet et al. 2016). Such an investigation has already been performed by Valle et al. (2014), Truitt et al. (2015), and more recently by Gallet et al. (2016), but in the case of non-rotating stellar models. Here we investigate for the first time the impact of stellar rotation and look for a correlation between the stellar activity and evolution of the HZ limits (hereafter HZLs). We also give more details and extend the results of Gallet et al. (2016).

The structure of this article is as follows. In Sect. 2 we present the reference grid of stellar models that we used to get the evolution of the internal structure and global parameters that play a role in the current prescriptions for habitability. In Sect. 3

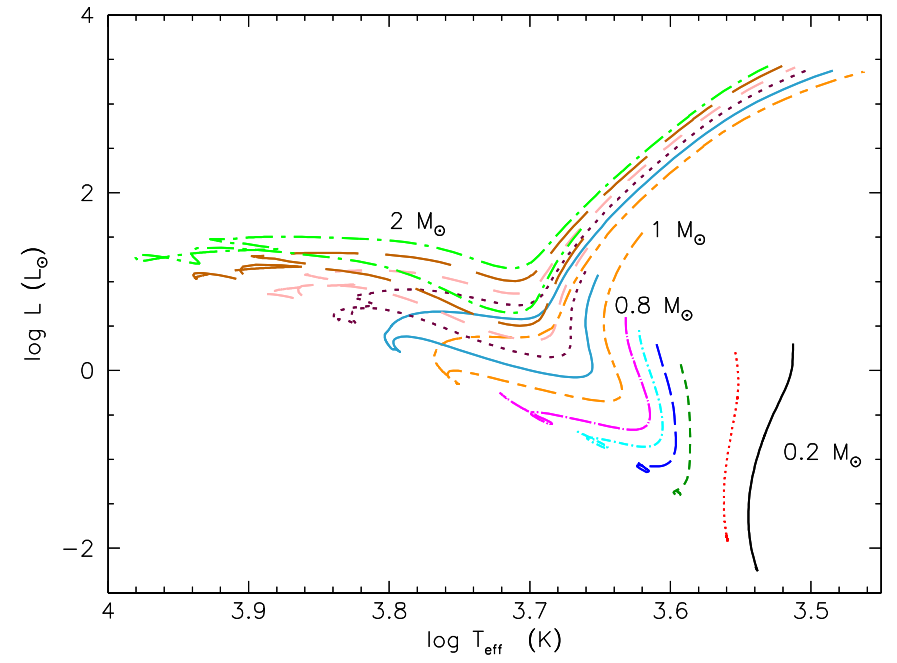

Fig. 1. Stellar evolution tracks in the HRD for the standard models of $0.2,0.3,0.5,0.6,0.7,0.8,1.0,1.2,1.4,1.6,1.8$, and $2 M_{\odot}$ at solar metallicity. The tracks are shown up to the RGB tip for the more massive stars that have a lifetime lower than $20 \mathrm{Gyr}$ and up to this age for the lower mass stars.

we give the prescriptions we use for the definition of the $\mathrm{HZ}$ and its evolution from the early pre-main-sequence (PMS) phase up to the red giant branch (RGB) as a function of stellar parameters. We first study the impact of stellar parameters (including rotation and metallicity ranging from $Z=0.0005$ to $Z=0.0255$ ) along the evolution of a solar-type star in Sect. 4 and explore the range of stellar mass between 0.2 and 2 solar masses in Sect. 5. We then examine the evolution of the continuously habitable zone in Sect. 6. Finally, in Sect. 7 we explore the correlation between stellar activity and evolution of the habitable zone limits and we conclude in Sect. 8.

\section{Reference grid of stellar models}

This paper is based on a grid of standard and rotating stellar models that we computed with the code STAREVOL for a range of initial masses between 0.2 and $2 M_{\odot}$ and for four values of $[\mathrm{Fe} / \mathrm{H}]=0.28,0,-0.49$, and -1.42 corresponding to metallicities $Z=0.0255,0.0134,4.3 \times 10^{-3}$, and $5 \times 10^{-4}$ respectively.

Amard et al. (2016) provide detailed description of the stellar evolution code STAREVOL and of the physical inputs (equation of state, nuclear reaction, and opacities) and the mechanisms that impact the internal transport of chemicals and angular momentum. We briefly examine the main points.

\subsection{Basic input physics}

At solar metallicity, the initial helium mass fraction and mixing length parameters, whose values are 0.2689 and 1.702 , respectively, are calibrated on a solar model with the solar mixture of Asplund et al. (2009). We kept the same value of the mixing length parameter for all the stellar models. Mass loss rate is applied from the first model on the PMS and estimated using the prescription of Cranmer \& Saar (2011).

Figure 1 shows the evolution tracks in the HertzsprungRussell diagram (hereafter HRD) of the standard models of masses $0.2,0.3,0.5,0.6,0.7,0.8,1.0,1.2,1.4,1.6,1.8$, and $2 M_{\odot}$ at solar metallicity. 
Table 1. Initial velocity used for the stellar rotating tracks at solar metallicity.

\begin{tabular}{ccc}
\hline \hline $\begin{array}{c}\text { Mass } \\
\left(M_{\odot}\right)\end{array}$ & $\begin{array}{c}\text { Initial velocity } \\
\left(\Omega_{\odot}\right)\end{array}$ & Designation \\
\hline 0.3 & 23 & fast \\
0.3 & 3.8 & slow \\
1.0 & 18.1 & fast \\
1.0 & 3.64 & slow \\
1.4 & 10 & fast \\
1.4 & 2.78 & slow \\
2.0 & 3.97 & fast \\
2.0 & 2.4 & slow \\
\hline
\end{tabular}

\subsection{Rotation}

In this work additional rotating models for the $0.3,1,1.4$, and $2 M_{\odot}$ stars (see Table 1) at various initial rotation rate are also considered but not shown in Fig. 1.

The treatment of angular momentum evolution is applied from the first model on the PMS phase using the complete formalism developed by Zahn (1992), Maeder \& Zahn (1998), and Mathis \& Zahn (2004), which takes into account advection by meridional circulation and diffusion by shear turbulence (see Palacios et al. 2003, 2006; Decressin et al. 2009). Solid body rotation is assumed for the convective regions. Magnetic braking is applied from the PMS onwards and up to the tip of the RGB following Matt et al. (2015).

The initial stellar rotation of the solar-like star model is calibrated using the two extreme rotational tracks (fast and slow) from the two-zone model of Gallet \& Bouvier (2015). For lower and higher masses the observed ratio $r=\Omega_{*} / \Omega_{\text {crit }}$ at $1 \mathrm{Myr}$, where $\Omega_{\text {crit }}$ is the critical velocity, is adopted to constrain the initial velocity, where $r=40 \%$ and $6 \%$ for the fast and slow rotators, respectively.

In addition to the treatment of transport and loss of angular momentum and mass, the models include the modification of the effective gravity by the centrifugal forces and its effect on the stellar structure equations following Endal \& Sofia (1976).

\section{Habitable zone}

\subsection{Definitions}

There are two methods to estimate the location of the HZLs. The first relies on the equilibrium temperature calculation that requires a strong assumption about the planetary Bond albedo, i.e. the reflectance of the surface of the planet that is usually taken equal to the Earth's albedo $=0.3$. The second method is based on a climate model, which directly estimates the albedo as the ratio between the upwards and downwards fluxes inside the planetary atmosphere that are emitted and received, respectively, by the planet (Kasting et al. 1993; Kopparapu et al. 2013, 2014).

The HZ of an exoplanet is generally defined as the area (in terms of distance from its central star) in which a rocky planet with the right atmosphere can maintain liquid water at its surface (see Kopparapu et al. 2013, 2014; Linsenmeier et al. 2015; Torres et al. 2015). Planets complying to this definition are named class I planets by opposition to other type of planets where liquid water can be found under an ice crust (see Lammer et al. 2009) with the possibility of a subsurface habitability. The HZLs are analytically given by Kasting et al. (1993) as

$d=\left(\frac{L / L_{\odot}}{S_{\text {eff }}}\right)^{0.5} \mathrm{AU}$,

where $d$ refers to both the inner or outer edge of the HZ (see below), $L$ is the stellar luminosity, $L_{\odot}$ the luminosity of the present Sun, and $S_{\text {eff }}$ is the effective stellar flux (Kasting et al. 1993; Kopparapu et al. 2013, 2014) expressed as the ratio of the outgoing IR flux, $F_{\text {IR }}$, produced by the planet to the net incident stellar flux, $F_{\text {inc }}$, received by the planet from the star

$S_{\text {eff }}=\frac{F_{\text {IR }}}{F_{\text {inc }}}$.

The parameter $F_{\mathrm{IR}}$ is estimated thanks to $1 \mathrm{D}$ radiativeconvective climate models such as that developed by Kasting et al. (1993) and Leconte et al. (2013b).

Usually, the inner edge of the HZ is chosen to correspond to the runaway greenhouse limit, which in turn corresponds to the moment when the surface temperature of the planet is high enough so that the surface of the planet and the base of its atmosphere start to radiate in both the near-IR and visible wavelengths. These radiations thus increase the outwards flux $F_{\text {IR }}$ while Rayleigh scattering effects, due to the vapour content, reduce the absorption of the near-IR flux inside the planetary atmosphere and lead to the saturation of the inwards flux $F_{\text {inc }}$ (Kopparapu et al. 2013, 2014). The surface temperature of the planet then reaches $T_{\text {surf }} \gg 647 \mathrm{~K}$ at which water reservoirs are completely evaporated. The legitimacy of this inner limit is presently questioned by the community as at that point where the surface temperature of a given planet is way too high to maintain Earth-like life. The runaway greenhouse limit represents an optimistic limit and somehow the extreme internal limit of the HZ. The outer edge of the $\mathrm{HZ}$ is defined by the maximum greenhouse limit, where the $\mathrm{CO}_{2}$ Rayleigh scattering reduces the efficiency of the greenhouse effect. At that limit, the planetary surface temperature is fixed at $273 \mathrm{~K}$ (i.e. the freezing point of water; Kasting et al. 1993; Selsis et al. 2007; Kopparapu et al. 2013, 2014).

In this work we refer to the HZLs as the location of the inner and outer edge of the $\mathrm{HZ}$ around the considered star, while the width of the $\mathrm{HZ}$ is the distance between the inner and outer edge of the HZ.

\subsection{Prescriptions from the literature}

In this section we describe different prescriptions from the literature that are used to estimate the HZLs. We compare in Sect. 4.2 the impact of each of these prescriptions on the HZLs.

\section{Kopparapu et al. (2014)}

Using the 1D radiative-convective, cloud-free climate model from Kasting et al. (1993), Kopparapu et al. (2014) provide an analytic expression for $S_{\text {eff }}$ that one can directly use to compute the HZLs. By using a polynomial fit, they expressed $S_{\text {eff }}$ as a function of the effective temperature of the host star $T_{\text {eff }}$ for different cases of HZLs definition (and thus planetary atmosphere) as

$S_{\text {eff }}=S_{\text {eff } \odot}+a T_{*}+b T_{*}^{2}+c T_{*}^{3}+d T_{*}^{4}$,

where $T_{*}=T_{\text {eff }}-5780 . S_{\text {eff } \odot}$ is self-consistently estimated and somehow represents the strength of the greenhouse effect. The 
Table 2. Value of the constants given by Kopparapu et al. (2014).

\begin{tabular}{cccccc}
\hline \hline & $S_{\text {eff॰ }}$ & $a\left(10^{-4}\right)$ & $b\left(10^{-8}\right)$ & $c\left(10^{-12}\right)$ & $d\left(10^{-15}\right)$ \\
\hline Inner & 1.107 & 1.332 & 1.58 & -8.308 & -1.931 \\
Outer & 0.356 & 0.6171 & 0.1698 & -3.198 & -0.5575 \\
\hline
\end{tabular}

Table 3. Value of the constants given by Selsis et al. (2007).

\begin{tabular}{cccc}
\hline \hline & $S_{\text {eff॰ }}$ & $a\left(10^{-4}\right)$ & $b\left(10^{-9}\right)$ \\
\hline Inner & $0.84-0.95$ & 0.27619 & 3.8095 \\
Outer & 1.67 & 1.3786 & 1.4286 \\
\hline
\end{tabular}

model parameters $S_{\text {eff } \odot}, a, b, c$, and $d$ depend on the planetary atmosphere. Here we use the values listed in Table 2.

For the inner edge, Kopparapu et al. (2013) started their modelling with a fully saturated "Earth" model and considered that the flux received by the planet at the top of its atmosphere is the same as that received from the Sun by the Earth (i.e. $\left.1360 \mathrm{~W} \mathrm{~m}^{-2}\right)$. Then they increased the surface temperature $\left(T_{\mathrm{s}}\right)$ of the planet from 200 up to $2200 \mathrm{~K}$ and looked at the evolution of the two fluxes, planetary albedo, and $S_{\text {eff }}$; this is the moment when $S_{\text {eff }}$ runaways define the runaway greenhouse limit. For the outer edge, Kopparapu et al. (2013) started their simulation with a 1 bar $\mathrm{N}_{2}$ planetary atmosphere with a surface temperature of $273 \mathrm{~K}$ (i.e. the freezing point of water). Then they increased the $\mathrm{CO}_{2}$ partial pressure from 1 to 35 bar and followed the evolution of $S_{\text {eff }}$, which exhibits a minimum that is reached when the efficiency of the greenhouse effect due to the $\mathrm{CO}_{2}$ compound is maximum. This minimum is called the maximum greenhouse limit and corresponds to the largest distance from the star that is required to maintain a surface temperature of $273 \mathrm{~K}$ on the planet.

Kopparapu et al. (2014) explored three different masses (0.1, 1 , and $5 M_{\oplus}$ ) and concluded that the larger the planet, the wider the HZLs. In the present work we only consider the Earth mass planet case.

All the calculations reported in Kopparapu et al. (2014) assumed a Sun-like star but these prescriptions are applicable, using interpolation, for stars with effective temperatures between 2600 and $7200 \mathrm{~K}$, i.e. between 0.1 and $1.4 M_{\odot}$ (Kopparapu et al. 2014).

Selsis et al. (2007)

Selsis et al. (2007) also provide a prescription for the inner and outer edge of the HZ that is based on the various cases of planetary atmosphere explored by Kasting et al. (1993). They use the following expression for the effective stellar flux:

$$
S_{\mathrm{eff}}=\frac{1}{\left(S_{\mathrm{eff} \odot}-a T_{*}-b T_{*}^{2}\right)^{2}},
$$

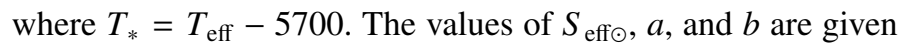
in Table 3. For the inner edge we used $S_{\text {eff } \odot \text {,in }}=0.84$ in the case of a runaway greenhouse limit $\left(0.95\right.$ corresponds to the $T_{\text {surf }}=$ 373 K limit).

The parabolic fitting of Selsis et al. (2007) is here scaled to the values found by Kasting et al. (1993) in the case of a G2 star and can be extrapolated to stars with $T_{\text {eff }}$ between 3700 and $7200 \mathrm{~K}$.
Table 4. Value of the constants given by Underwood et al. (2003).

\begin{tabular}{cccc}
\hline \hline & $S_{\text {eff }}$ & $a\left(10^{-4}\right)$ & $b\left(10^{-8}\right)$ \\
\hline Inner & 1.268 & -2.139 & 4.190 \\
Outer & 0.2341 & -0.1319 & 0.619 \\
\hline
\end{tabular}

Underwood et al. (2003)

We finally give the $\mathrm{HZ}$ prescription from Underwood et al. (2003), who also use a polynomial fit to express the effective stellar flux $S_{\text {eff }}$,

$S_{\text {eff }}=S_{\text {eff } \odot}+a T_{\text {eff }}+b T_{\text {eff }}^{2}$,

where $T_{\text {eff }}$ is the effective stellar surface temperature and the values of $S_{\text {eff } \odot}, a$, and $b$ are summarised in Table 4.

Using the different cases provided by Kasting et al. (1993), Underwood et al. (2003) estimate the effective stellar flux as a function of the effective temperature between $3700 \mathrm{~K}$ (M0 star) and $7200 \mathrm{~K}$ (F0 star).

Among the three expressions above, Selsis et al. (2007) and Underwood et al. (2003) are both based on the work of Kasting et al. (1993) and the difference that they may have is a consequence of the fitting method employed to express the stellar flux $S_{\text {eff }}$ as a function of $T_{\text {eff }}$. The main difference between Kopparapu et al. (2014) and the other two prescriptions lies in an update of the Kasting et al. (1993) model by Kopparapu et al. (2014). This update includes the most recent version of the HITEMP opacity database and additional $\mathrm{H}_{2} \mathrm{O}$ lines in the nearinfrared (Kopparapu et al. 2013). The impact of these prescriptions are studied in Sect. 4.2. After this comparison, we use the Kopparapu et al. (2014) prescription.

\subsection{Limitations of the current prescriptions for $\mathrm{HZ}$}

The prescriptions described above are very sensitive to the climate model used to estimate the impact of the incident stellar flux. Therefore, they are only valid in the framework of the specific climate model developed by Kasting et al. (1993). Moreover, the atmospheric compositions used by Kopparapu et al. (2014) imply that their HZ prescriptions are only valid in the case of a planetary atmosphere similar to that of the Earth at the present time. This corresponds to the main-sequence (MS) phase of our Sun, where the HZ and stellar parameters are not evolving much and where the stellar activity is low. However, the atmospheric composition of the Earth has drastically changed during the last 4 Gyr. It is thought that this latter reached its current composition between 400 to $600 \mathrm{Myr}$ ago. In this work we provide the entire $\mathrm{HZ}$ evolution anyway to highlight the fact that stellar models should be used, especially, during the initial phases of stellar evolution during which stellar parameters heavily and clearly evolve. However, the results need to be taken with caution, as the evolution of the planetary atmosphere must be taken into account as well, and a complete modelling should involve the co-evolution of both the star and the planetary atmosphere (Leconte et al. 2013a).

These prescriptions are also dedicated to stars with effective temperatures between 2600-3700 and $7200 \mathrm{~K}$ (Underwood et al. 2003; Selsis et al. 2007; Kopparapu et al. 2014) corresponding to stellar mass of 0.1 and $1.4 M_{\odot}$, respectively. Kasting et al. (1993) and Kopparapu et al. (2014) chose to stop their analysis at this upper mass limit because stars beyond $1.5 M_{\odot}$ have a lifetime that is shorter than 2 Gyr while it is thought that complex 
life needs more than 1 Gyr to evolve. We extrapolated these prescription for higher mass star, however, to obtain a large range of initial stellar mass. The validity of this extrapolation is of course questionable, but in the following we show that the evolution of the $\mathrm{HZ}$ of a $2 M_{\odot}$ star is consistent (same temporal features) with that of a $1 M_{\odot}$ star. Moreover, the habitable zone distance $d$ (Eq. (1)) have a weak dependence on $T_{\text {eff }}$ (through $S_{\text {eff }}$ ). For instance, a change of $20 \%$ of $S_{\text {eff }}$ only modifies $d$ by about $10 \%$.

Finally, all these quantities are given in the case of a cloud free atmosphere. Selsis et al. (2007) studied the impact of the presence of cloud (from a cloud free to a planet fully covered by cloud, using the Kasting et al. (1993) model) on the inner and outer edge of the HZ (see their Table 2). While the outer edge of the HZ is not impacted by the percentage of cloud, the inner edge is reduced almost by a factor of two when the covering percentage goes from $0 \%$ to $100 \%$.

\section{Evolution of the habitable zone: the case of a $1 M_{\oplus}$ planet around an evolving $1 M_{\odot}$ host star}

In this section, we focus on the case of a $1 M_{\oplus}$ planet around a $1 M_{\odot}$ mass star and study the impact of the intrinsic stellar evolution, metallicity, and rotation on the HZLs. We do not include the effects of planetary mass since they have been shown to be relatively marginal (at least for planets with masses below $5 M_{\oplus}$; see Kopparapu et al. 2013, 2014).

\subsection{Generalities}

The HZLs evolution around a $1 M_{\odot}$, non-rotating, star with solar metallicity can be followed in Fig. 2 where we show the temporal evolution of the inner and outer edges of the HZ from the early PMS to the tip of RGB. This evolution can be separated into several main phases: PMS, zero-age main sequence (ZAMS), MS, and post-main sequence (e.g. sub-giant phase and RGB).

The PMS phase starts along the so-called Hayashi track (Hayashi 1961), where the star evolves at nearly constant effective temperature and decreasing surface luminosity. One first sees a rapid and sharp decrease of the size of the HZLs that reach a minimum at the end of the Hayashi track (i.e. between $2 \times 10^{2}$ and $1.5 \times 10^{7} \mathrm{yr}$ ). The PMS phase continues along the Henyey track (for stars above $0.5 M_{\odot}$, Henyey et al. 1955), where the star evolves at nearly constant luminosity and increases effective temperature towards the ZAMS; the ZAMS is defined by the moment during the evolution when hydrogen burning is dominant in the stellar core, i.e. when the hydrogen abundance has dropped to about 0.998 of the initial abundance. This is reached at about $50 \mathrm{Myr}$ for our solar model. During this last PMS stage, the HZLs slightly increase and reach a local maximum at ZAMS.

At the ZAMS the stellar structure eventually reaches a quasisteady state because of a quasi-hydrostatic equilibrium regime that is ensured by efficient nuclear reactions. At that point the internal temperature of the star is high enough to initiate complex nuclear reactions, which then counteract the stellar contraction by balancing the gravity with thermal pressure. During the MS phase (i.e. from 50 Myr to about $10 \mathrm{Gyr}$ ) the HZLs remain more or less constant at about 1.5 AU for the outer edge and $0.8 \mathrm{AU}$ for the inner edge (Gallet et al. 2016). This confirms the general assumptions of a relative constancy of these limits during the MS phase.

Finally, during the RGB phase (i.e. from about $10 \mathrm{Gyr}$ ) the star evolves towards higher luminosity and lower effective

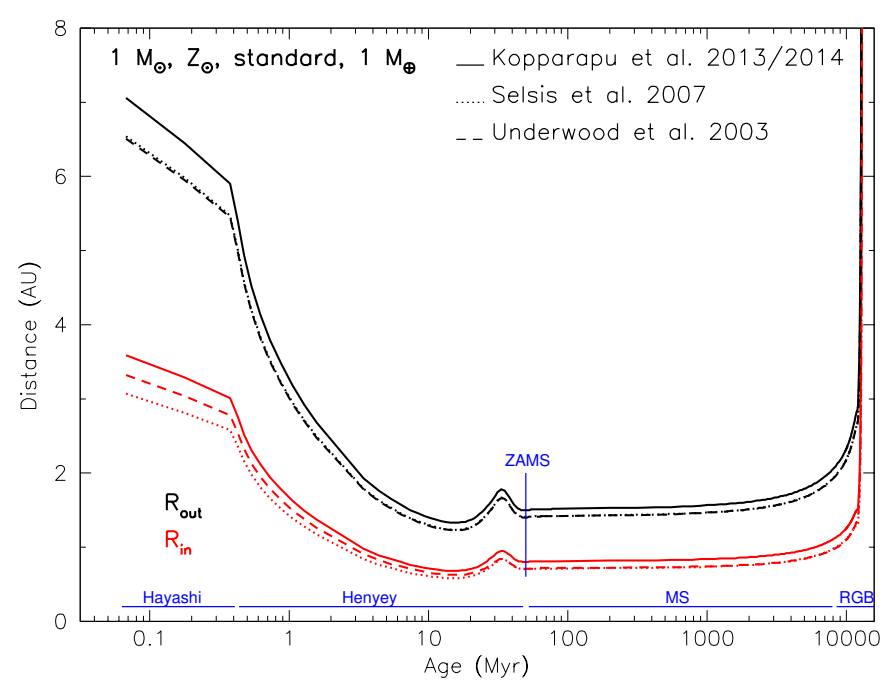

Fig. 2. Evolution of the HZLs as a function of age for a $1 M_{\odot}$ star with solar metallicity. The inner and outer edge of the $\mathrm{HZ}$ are represented by the red and black lines, respectively. The solid, dotted, and dashed lines are associated with the HZ prescription from Kopparapu et al. (2014), Selsis et al. (2007), and Underwood et al. (2003), respectively. The blue lines depict the localisation of the different phases: PMS (Hayashi, Henyey), ZAMS, MS, and RGB.

temperature onwards. Correspondingly, the HZLs are shifted outwards reaching values as high as $8 \mathrm{AU}$ to $12 \mathrm{AU}$. During this final stage, the HZLs move continuously away from the star, whose luminosity increases.

The respective durations of each evolutionary phase are clearly unbalanced and have different kinds of effects on the evolution of life itself. For instance, while a $1 M_{\odot}$ star passes only about 50 Myr at the PMS phase, it will pass several Gyr at the MS phase. Hence, the PMS phase is clearly too short to be considered as relevant for the development of life. However, to constrain the planetary habitability of a given planet, this very early phase plays a key role through the MHD and tidal star-planet interaction (see Zahn \& Bouchet 1989; Vidotto et al. 2013; Strugarek et al. 2014, 2015; Mathis 2015; Mathis et al. 2016, and Sect. 7 of this article). Furthermore, as the HZLs sharply evolve during the PMS phase, the surface conditions of a planet could also strongly evolve and thus later constrain the framework in which life evolves.

\subsection{Impact of the $H Z$ prescription}

In Fig. 2 we also compare the HZLs prescriptions from Underwood et al. (2003), Selsis et al. (2007), and Kopparapu et al. (2013, 2014). Since all these prescriptions have a common base, the shape of the HZLs evolution is the same for the three prescriptions. Kopparapu et al. (2014) produces higher values for the inner (hereafter $R_{\text {in }}$ ) and outer (hereafter $R_{\text {out }}$ ) edge of the HZ compared to Selsis et al. (2007) and Underwood et al. (2003). While there are no significant differences between Selsis et al. (2007) and Underwood et al. (2003) for $R_{\text {out }}$, there is a small shift towards lower values for $R_{\text {in }}$ from the PMS to the MS phase for the Selsis et al. (2007) prescription compared to Underwood et al. (2003).

The prescriptions of Selsis et al. (2007) and Underwood et al. (2003) are both based on the HZ estimations given in Kasting et al. (1993) for three stellar effective temperatures $(3700,5700$, and $7200 \mathrm{~K}$; see Table 3 from Kasting et al. 1993). The difference in fitting method between 


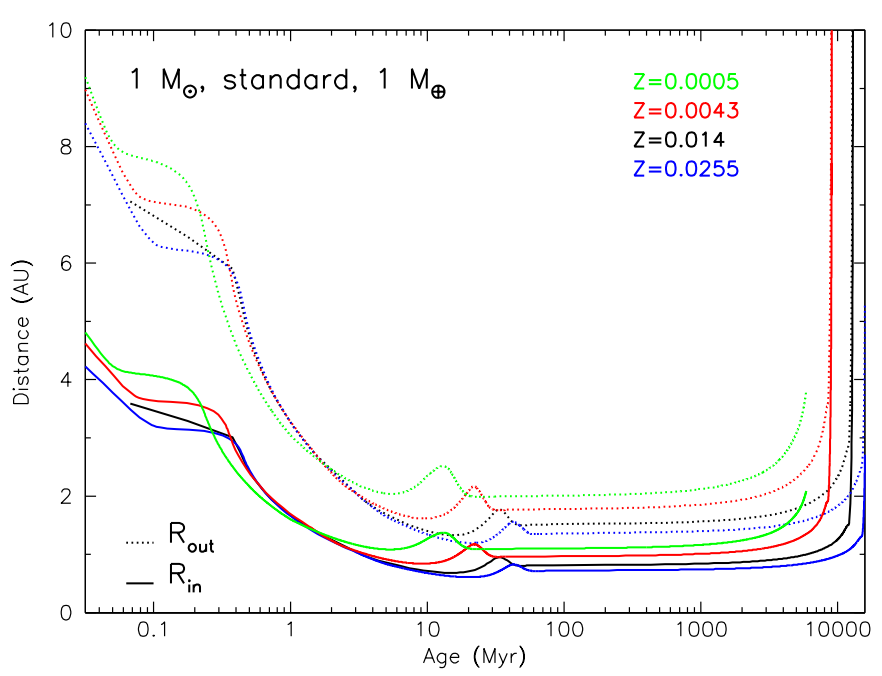

Fig. 3. Evolution of the $\mathrm{HZ}$ as a function of the stellar age for a $1 M_{\odot}$ star and for metallicities $Z=0.0255,0.014,0.0043$, and 0.0005 (blue, black, red, and green, respectively). The inner and outer edge of the HZ are represented by solid and dashed lines, respectively.

the three HZ prescriptions leads, for the inner edge calculated with Underwood et al. (2003), to a higher $R_{\text {in }}$ at low temperature and to a lower $R_{\text {in }}$ at high temperature compared to Selsis et al. (2007). Conversely, for the outer edge calculated with Underwood et al. (2003), this leads to a lower $R_{\text {out }}$ at low temperature and to a higher $R_{\text {out }}$ at high temperature compared to Selsis et al. (2007) (see Fig. 2).

Therefore, as shown by Fig. 2 the impact of the fitting method on the HZ is non-negligible, and even if these prescriptions have a common base (i.e. Kasting et al. 1993) these methods lead to slightly different estimates of the HZLs of about $10 \%$.

\subsection{Metallicity effect}

Metallicity is one of the main ingredients that strongly change the structure and evolution of a star of a given initial mass.

The major effect of metallicity is to produce, owing to opacity effects, a translation in both luminosity and effective temperature along the evolution tracks in the HRD. When the metallicity decreases, the quantity of metallic bound-free absorption inside the star is reduced. This leads to the decrease of the stellar opacity allowing an easier escape of energy and thus increasing the stellar luminosity and temperature. For a given initial stellar mass and at a given evolutionary phase, $T_{\text {eff }}$ and $L_{*}$ both increase for decreasing metallicity. This directly produces an increase of the HZLs that almost evolves as $L_{*}^{0.5}$ (since the HZLs do not depend much on the effective temperature according to Kopparapu et al. 2013, 2014). Figure 3 shows the evolution of the HZLs in the case of a $1 M_{\odot}$ star for $[\mathrm{Fe} / \mathrm{H}]$ ranging between $+0.28 \operatorname{dex}(Z=0.0255)$ and $-1.42 \operatorname{dex}(Z=0.0005)$.

In addition to a shift in luminosity and effective temperature, changing the stellar metallicity also modifies the time at which the characteristic features on the tracks appear. Indeed, the overall lifetime of a star decreases for decreasing metallicity.

As shown in Gallet et al. (2016), the impact of metallicity is clearly visible at ZAMS. First, the location of the "bump" (see Sect. 5) is shifted towards younger ages for decreasing metallicities (see above). As a result of faster evolution, the maximum amplitude of the "bump" is reached earlier for decreasing metallicity. Moreover, a decrease in metallicity moves the inner and outer edge of the HZ outwards.

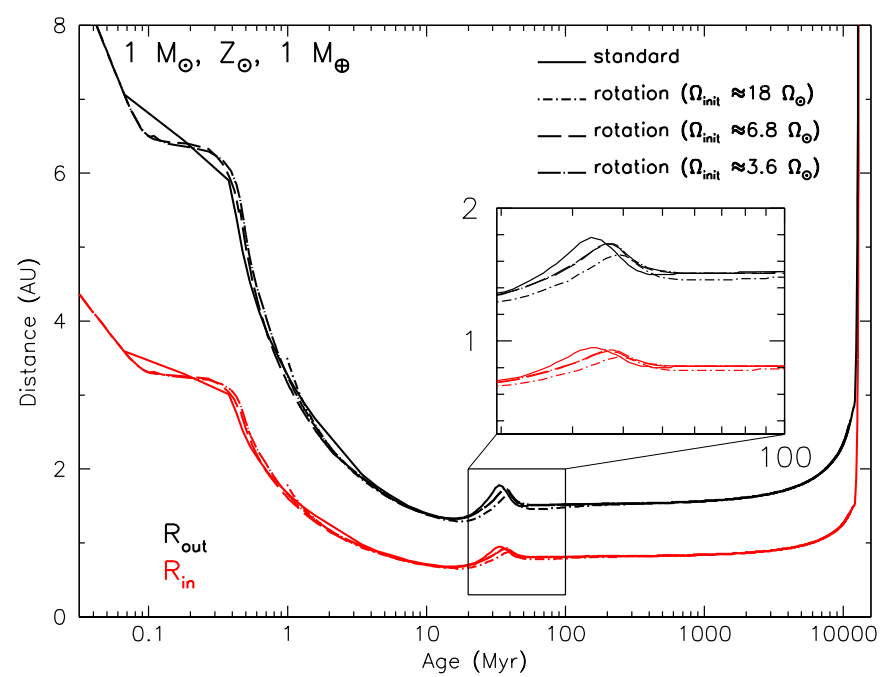

Fig. 4. Effect of the change of initial angular velocity on the evolution of the HZLs in the case of a $1 M_{\odot}$ star with solar metallicity. Inner and outer edge of the habitable zone are represented by red and black lines, respectively. Standard model is plotted in dotted line and rotation models in dash-dotted, long-dashed, and long-dash-dotted lines for 18 , 6.8 , and $3.6 \Omega_{\odot}$ initial rotation rate, respectively.

\subsection{Impact of stellar rotation}

A star is naturally a rotating body. From its formation inside a molecular cloud up to its nuclear death, the rotation always plays a central role (Maeder 2009). Stellar rotation modifies the stellar effective temperature and luminosity, which are important parameters for the location of the HZ. It also has an indirect impact on the HZ through stellar activity (see Sect. 7). While the evolution of stellar angular velocity is now fairly well known (Reiners \& Mohanty 2012; Gallet \& Bouvier 2015; Johnstone et al. 2015; Amard et al. 2016), the early-PMS phase and its star-disk interaction (hereafter, SDI) remain a mystery (Ferreira 2013). Yet, planetary formation is thought to happen during the first 10 Myr (Bell et al. 2013) of the lifetime of the disk, which may be interrupted by the SDI and then have severe consequences on the first stage of planetary evolution.

The effective surface temperature and luminosity of a given star slightly decreases with increasing rotation rate; there are also marginal effects on lifetime of a star, which is slightly reduced with increased stellar rotation. Figure 4 shows the evolution of the corresponding HZLs for standard and rotating models. This figure shows that the effect of a change in rotation rate on the HZLs is marginal (the four lines are almost superimposed). The maximum effect of rotation is seen at ZAMS. Indeed, as the initial rotation rate of given rotating model increases, the corresponding star reaches an higher angular velocity at the end of the contraction phase where the rotation rate reaches a maximal value (see Gallet \& Bouvier 2013, 2015; Amard et al. 2016). As centrifugal effects are directly proportional to the square of the stellar angular velocity, they have maximum impact during this phase. This effect decreases as the initial rotation rate decreases since the star tends to reach the standard model case when reducing its rotation rate.

\subsection{Impact of the microphysics used in stellar models}

Valle et al. (2014) and Truitt et al. (2015) also analyse the impact of stellar parameters on the evolution of the HZLs. While Valle et al. (2014) based their analysis on the FRANEC code 
F. Gallet et al.: Impacts of stellar evolution and dynamics on the habitable zone: The role of rotation and magnetic activity

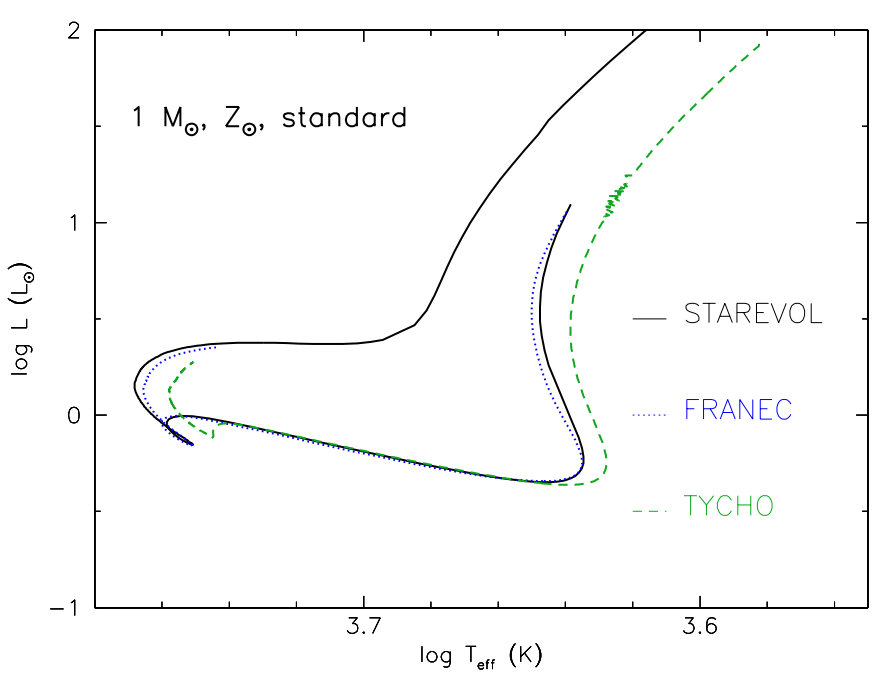

Fig. 5. Stellar evolution tracks in the HRD for the standard models of a $1 M_{\odot}$ at solar metallicity. The black solid, blue dotted, and dark green dashed lines are associated with stellar models from this work, Valle et al. (2014), and Truitt et al. (2015), respectively.

(Degl'Innocenti et al. 2008), Truitt et al. (2015) used the TYCHO code (Young \& Arnett 2005) to calculate the evolution of both luminosity and effective temperature. In this section, we compare the impact of the corresponding choice of input microphysics on the evolution of the HZLs.

Figure 5 shows the Hertzsprung-Russel diagram of a $1 M_{\odot}$ star with solar metallicity from the STAREVOL, FRANEC, and TYCHO stellar evolution codes, respectively. The microphysics used in these last two stellar codes are briefly recalled here.

\section{TYCHO: Young \& Arnett (2005)}

$\mathrm{TYCHO}^{2}$ and STAREVOL contain different microphysics in terms of chemical mixture, opacities, and nuclear reactions. With the available models, however, it is not possible to precisely isolate the impact of each of these changes. For this model the chemical mixture of Lodders (2010) was used with a metallicity $Z=0.0153$ and an initial helium mass fraction $Y=0.2735$.

\section{FRANEC: Tognelli et al. (2011)}

This latest version of the FRANEC $^{3}$ code has been upgraded and now includes new abundances and atmospheric conditions. Even if different mixing length parameters are used between STAREVOL and FRANEC (1.702, and 1.68, respectively), the results of these models are close to each other as shown by Fig. 5 . For the FRANEC model, the chemical mixture of Asplund et al. (2005) was used with a metallicity $Z=0.01377$ and an initial helium mass fraction $Y=0.2533$.

As shown by Fig. 5 the difference of metallicity between TYCHO and STAREVOL/FRANEC results in an cooler stars (at a given luminosity) for TYCHO compared to STAREVOL/FRANEC. As the dependence of the HZLs on the effective temperature is small (see Kopparapu et al. 2013, 2014),

\footnotetext{
2 TYCHO Interpolation Tool, nicknamed Uranienborg, http://bahamut. sese.asu.edu/ payoung/AST_522/ Evolutionary_Tracks_Database.html

3 FRANEC, http://astro.df.unipi.it/stellar-models/ index. $p h p ? m=8$
}

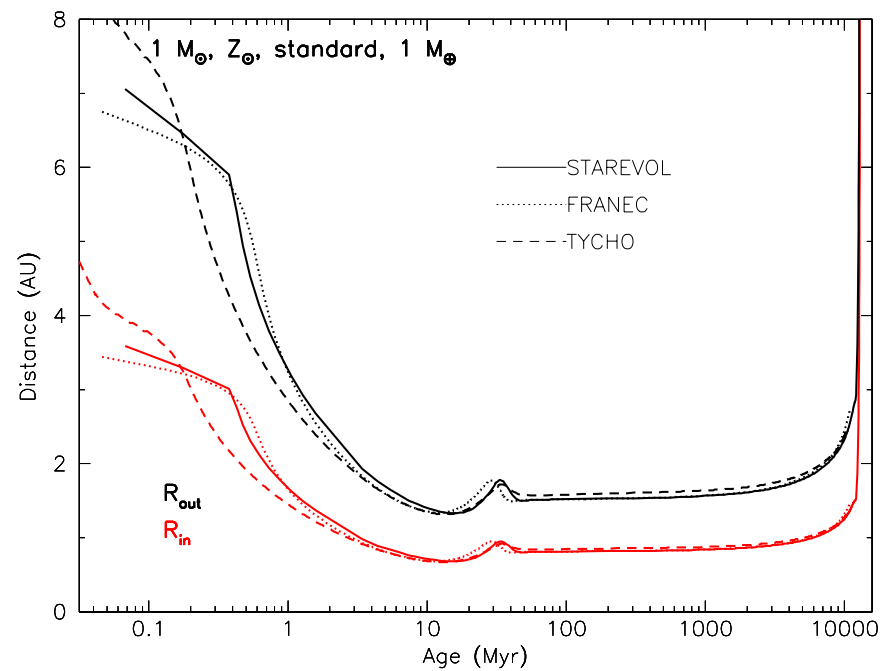

Fig. 6. Evolution of the HZLs as a function of age for a $1 M_{\odot}$ with solar metallicity. The inner and outer edge of the $\mathrm{HZ}$ are represented by the red and black lines, respectively. The solid, dotted, and dashed lines are associated with stellar models from this work, Valle et al. (2014), and Truitt et al. (2015), respectively.

the evolution of the HZLs related to these three stellar codes is expected to be relatively similar (see Fig. 6.)

\subsection{Summary}

In order to analyse the impact of stellar parameters and stellar models on the evolution of the HZ, we focused on an Earth-mass planet orbiting a solar-type star. We first reviewed the impact of numerical modelling considerations, such as the choice of stellar model, to best estimate the location of the HZ. Then, we evaluated the impact of a change in the stellar physical parameters on the evolution of the inner and outer edge of the HZ.

As shown, a change of $\mathrm{HZ}$ prescription has a small impact on the HZLs. The HZ prescription of Selsis et al. (2007) and Underwood et al. (2003) are on average around $10 \%$ closer to the star compared to the HZ prescription of Kopparapu et al. (2014).

From the point of view of the physical stellar properties, we showed that a change, even small, in metallicity dramatically impacts the location of the HZ, especially beyond the ZAMS and during the entire MS phase. A decrease in metallicity moves the $\mathrm{HZ}$ outwards (because $L_{*}$ and $T_{\text {eff }}$ both increase) and shortens the stellar evolutionary phases. With a change of metallicity of almost $100 \%(Z=0.0255$ to $Z=0.0005)$ the inner and outer edge moved outwards, at ZAMS, by about $50 \%$. Rotation marginally affects the $\mathrm{HZ}$ and its effects are the strongest when the stellar rotation is maximum, that is at the end of the contraction phase. During this phase, the effect of rotation is only to move the HZ closer to the star of about $10 \%$ and will only last few tenths of Myr in the case of a solar-type star.

The impact of microphysics input is not null but marginal and only affects the earliest phase of stellar evolution, namely the PMS phase (see Fig. 6). Indeed, during the most important phase in terms of duration (i.e. the MS) the input microphysics only has a minor impact as long as the luminosity during that phase is not much affected. The TYCHO and FRANEC stellar evolution code produced at ZAMS an HZ that is about 3 and $1 \%$ closer to the star compared to the STAREVOL code. However, a more detailed analysis should be performed so as to extract the specific impact of each of these input microphysics. 
Table 5. Size of the $\mathrm{HZ}$ as a function of stellar mass.

\begin{tabular}{ccccc}
\hline \hline$\Delta H Z$ & $0.3 M_{\odot}$ & $1 M_{\odot}$ & $1.4 M_{\odot}$ & $2 M_{\odot}$ \\
\hline$\Delta H Z_{\text {mean }}(\mathrm{AU})$ & 0.13 & 0.86 & 1.68 & 3.25 \\
$\Delta H Z_{\min }(\mathrm{AU})$ & 0.12 & 0.65 & 1.08 & 1.85 \\
$\Delta H Z_{\max }(\mathrm{AU})$ & 0.48 & 3.46 & 5.45 & 6.63 \\
\hline
\end{tabular}

\section{Habitable zone of a $1 M_{\oplus}$ planet around stars of various initial masses}

In this section we extend the previous study to evaluate the impact of the stellar mass on the HZLs along stellar temporal evolution.

Figure 1 shows that, at a given evolution the phase, luminosity, and effective temperature increase for increasing mass. The direct effect of a mass increase on the HZLs is to shift both the inner and outer edges of the HZ further away from the star and to increase the width of the HZ.

Figure 7 shows the evolution of $R_{\text {in }}$ and $R_{\text {out }}$ as a function of time and stellar mass for all the models at solar metallicity $Z=0.0134$. As shown in Gallet et al. (2016), the evolution of $R_{\text {in }}$ and $R_{\text {out }}$ along the PMS depends on the stellar mass. During that phase, these quantities continuously decrease for lowmass stars $\left(M_{*}<0.6 M_{\odot}\right)$, while for higher mass stars they first decrease before increasing when $\mathrm{CNO}$ burning reaches equilibrium as the stars approach the ZAMS. Then for all stellar masses, $R_{\text {in }}$ and $R_{\text {out }}$ remain approximately constant along the MS before strongly increasing when the stars move towards and along the RGB.

The increase of HZ is not linear with the variation of mass, i.e. at about 100-200 Myr, the difference between $R_{\text {in }}$ or $R_{\text {out }}$ of stars separated by $0.1 M_{\odot}$ strongly increases with mass, and the width of the HZ increases for increasing stellar mass as well. On average, and for a non-rotating star with solar metallicity, the width of the $\mathrm{HZ}$ is about $0.13 \mathrm{AU}$ for a $0.3 M_{\odot}$ star and $3.25 \mathrm{AU}$ for a $2 M_{\odot}$ star. The variation on stellar mass also dramatically affects the location of the HZ. At ZAMS, and by increasing the stellar mass from 1 to $2 M_{\odot}$, the HZLs moves outwards by more than $400 \%$.

Table 5 and Fig. 8 show the maximum, minimum, and averaged value of the width of the $\mathrm{HZ}$, where

$\Delta H Z_{\text {mean }}=\sum_{i=1}^{n} \frac{R_{\mathrm{out}, i}-R_{\mathrm{in}, i}}{n}$,

with $n$ the total number of steps from the stellar model. The minimum is reached at $352,14,7.35$, and $3.5 \mathrm{Myr}$, respectively for $0.3,1.0,1.4$, and $2.0 M_{\odot}$. The fact that the width of the $\mathrm{HZ}$ of high-mass stars is, on average, wider than that of low-mass stars suggests that the probability of a planet to be found inside the HZ is higher for higher mass stars. However, on the other hand, the duration of the continuously habitable zone strongly decreases for increasing stellar mass (see Sect. 6), which dramatically decreases the chance of a planet inside the $\mathrm{HZ}$ of a high-mass star to end up with a complex life. The effect of a change of stellar mass on the stellar temporal features is somehow the same as a change of metallicity; increasing mass and decreasing metallicity shortens the stellar life.

\section{Continuously habitable zone}

The continuously habitable zone (CHZ) is a region within the $\mathrm{HZ}$ where a planet, given its orbital radius, stays a minimum amount
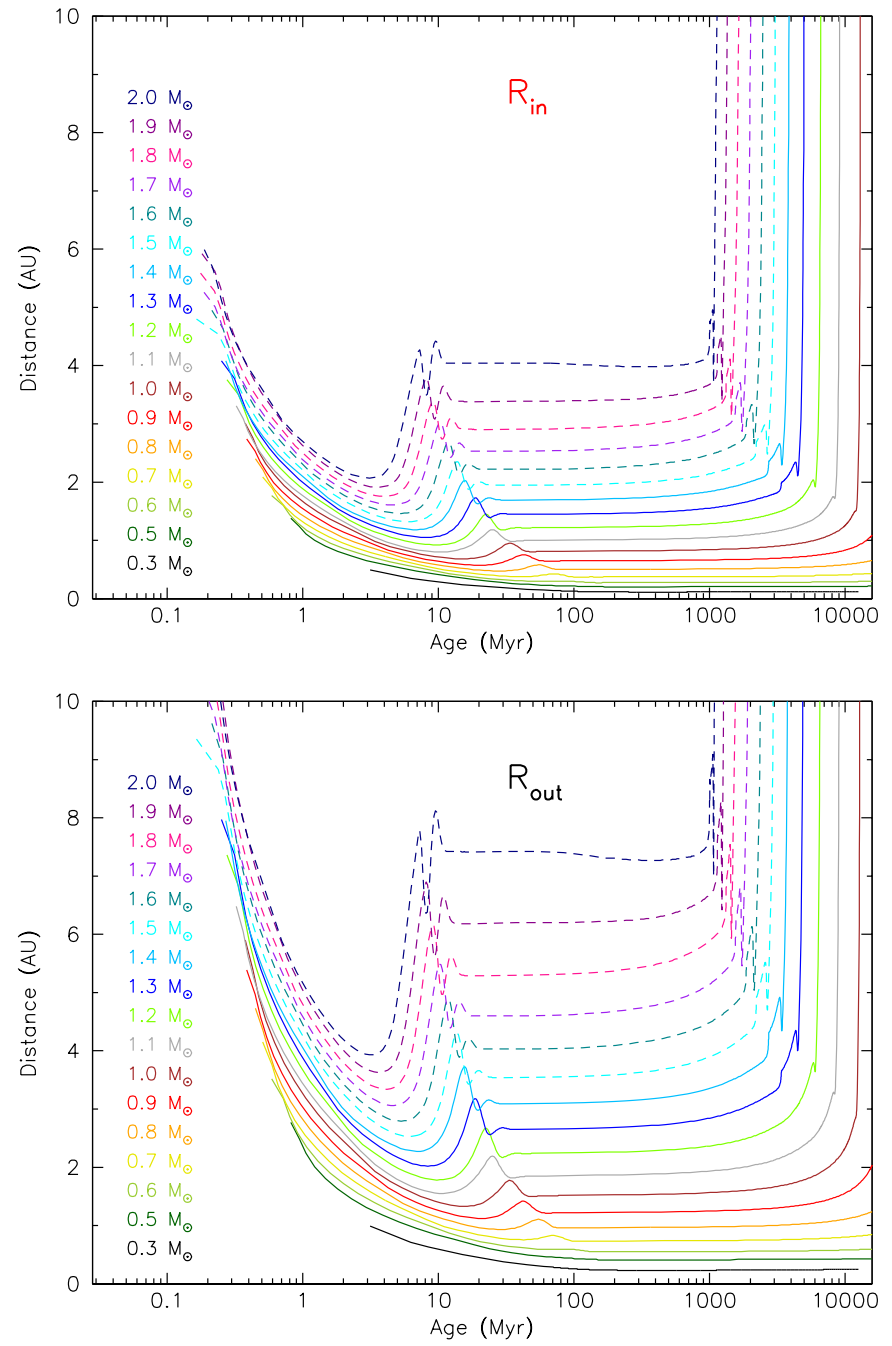

Fig. 7. Evolution of the inner edge and outer edges of the HZ (top and bottom, respectively) as a function of the time for stars of 0.3 (black), 0.5 (dark green), 0.6 (yellow-green), 0.7 (gold), 0.8 (orange), 0.9 (red), 1.0 (brown), 1.1 (grey), 1.2 (chartreuse), 1.3 (blue), 1.4 (deep sky blue), 1.5 (cyan), 1.6 (turquoise), 1.7 (purple), 1.8 (pink), 1.9 (magenta), and 2 (navy) $M_{\odot}$ with $Z_{\odot}$. Dashed lines represent stars outside of the theoretical range of application of the Kopparapu et al. (2014) prescription.

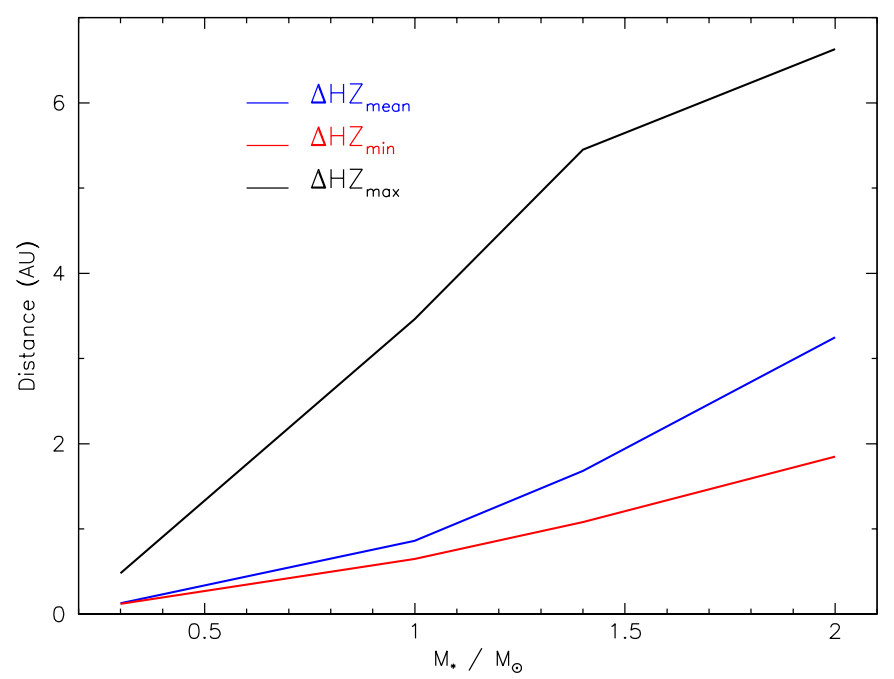

Fig. 8. Averaged (blue), minimum (red), and maximum (black), size of the $\mathrm{HZ}$ as a function of stellar mass. These values are compiled over the whole stellar evolution. 
F. Gallet et al.: Impacts of stellar evolution and dynamics on the habitable zone: The role of rotation and magnetic activity
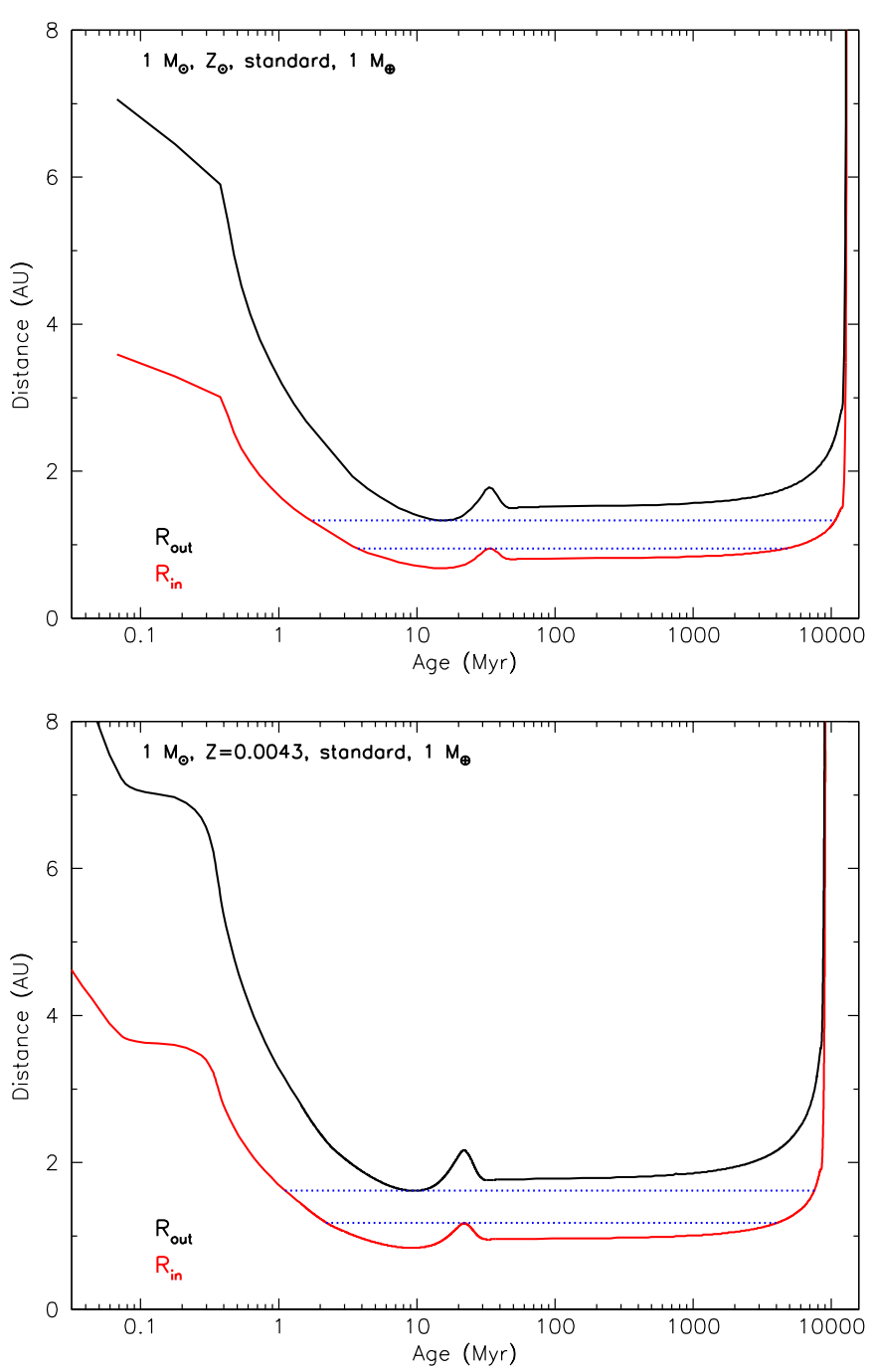

Fig. 9. Continuously habitable zone in the case of a $1 M_{\odot}$ star with solar metallicity and $Z=0.0043$ (top and bottom panels, respectively). The inner (red) and outer (black) HZLs were obtained using standard model and the Kopparapu et al. (2014) prescription. The blue horizontal lines delimit the area that corresponds to the maximum duration of a given planet within the HZ.

of time. This duration is generally assumed to lie between $2 \mathrm{Gyr}$ (Truitt et al. 2015) and 4 Gyr (Valle et al. 2014). According to Truitt et al. (2015), 2 Gyr is the time required by primordial life form on Earth to significantly alter the atmospheric composition of the planet so that this alteration (i.e. called biosignature) could be detectable if viewed from another planetary system (Kasting et al. 1993). On the other hand, 4 Gyr is thought to be the typical timescale for the emergence of complex life such as on Earth. However, these two timescales are somehow too restraining since they refer to an Earth-like life. Therefore, in the following we do not fix any minimum duration for the $\mathrm{CHZ}$, but rather simply look for the HZLs in which a potential planet stays the longest amount of time.

Figure 9 shows the evolution of the $\mathrm{HZ}$ as a function of time for two metallicities $\left(Z_{\odot}\right.$ and $Z=0.0043$, top and bottom panels respectively). The $\mathrm{CHZ}$ is depicted by the dotted blue lines in these figures. It corresponds to the region (expressed in astronomic units) inside the $\mathrm{HZ}$ where a planet potentially stays a maximum amount of time. Since we did not impose any minimum duration for a planet to be inside the $\mathrm{CHZ}$ in this work, we
Table 6. Size of the $\mathrm{CHZ}$ as a function of stellar mass for solar metallicity.

\begin{tabular}{ccccc}
\hline \hline Mass & $R_{\text {in }}(\mathrm{AU})$ & Time $_{\text {in }}(\mathrm{Gyr})$ & $R_{\text {out }}(\mathrm{AU})$ & Time $_{\text {out }}(\mathrm{Gyr})$ \\
\hline $0.3 M_{\odot}$ & 0.12 & $>20$ & 0.23 & $>20$ \\
$1 M_{\odot}$ & 0.95 & 4.77 & 1.33 & 10.67 \\
$1.4 M_{\odot}$ & 1.96 & 2.15 & 2.27 & 2.73 \\
$2.0 M_{\odot}$ & 4.27 & 0.99 & 7.0 & 1.09 \\
\hline
\end{tabular}

Notes. Time $(\mathrm{Gyr})=$ duration of the $\mathrm{CHZ}$ near the inner $\left(\mathrm{Time}_{\text {in }}\right)$ and outer $\left(\right.$ Time $_{\text {out }}$ ) edge of the HZ.

Table 7. Size of the CHZ as a function of planetary mass around a solar mass star with solar metallicity.

\begin{tabular}{ccccc}
\hline \hline Planet & $R_{\text {in }}(\mathrm{AU})$ & Time $(\mathrm{Gyr})$ & $R_{\text {out }}(\mathrm{AU})$ & Time $(\mathrm{Gyr})$ \\
\hline $0.1 M_{\oplus}$ & 0.92 & 4.88 & 1.33 & 11.01 \\
$1 M_{\oplus}$ & 0.95 & 4.77 & 1.33 & 10.67 \\
$5 M_{\oplus}$ & 1.01 & 4.88 & 1.33 & 10.07 \\
\hline
\end{tabular}

Notes. Time $(\mathrm{Gyr})=$ duration of the CHZ.

also give the potential time that a planet, located either close to the inner or outer edge of the CHZ, passes inside the CHZ.

For a solar-type star, the CHZ limits (CHZLs) move closer from the star for increasing metallicity (see Fig. 9). As the stellar lifetime decreases when decreasing the metallicity (see Sect. 4.3) the CHZLs also lasts longer for metal-rich stars. The time spent within the $\mathrm{CHZ}$ is longer for planets close to the outer edge of the $\mathrm{HZ}$ and decreases at lower metallicity. However, the width of the CHZLs (i.e. the size between the inner and outer edges of the CHZ) increases with decreasing metallicity. Moreover, stars with lower metallicity enter the $\mathrm{CHZ}$ earlier.

For a comparison purpose, we also listed the results for a 5 and $0.1 M_{\oplus}$ planets around a $1 M_{\odot}$ and $Z_{\odot}$ star in Table 7. The planetary mass only has a small effect on the location of the CHZLs and the duration of the CHZ. However there is small trend for smaller CHZLs and smaller duration of the outer CHZ towards heavier planets.

\section{Stellar activity, magnetospheric protection, and habitable zone}

Stellar and planetary magnetic fields are thought to be generated by dynamo action within their convective layers (e.g. Stevenson 2003; Brun et al. 2015b). On the one hand, in the case of the star, the magnetic field plays a major role in its overall dynamics and shapes its environment. The magnetic field is also involved in SDI processes, stellar winds and magnetic braking, and surface activity (starspots, flares, and CMEs) and, as a consequence, has a direct impact on the surrounding planets. On the other hand, planetary magnetic fields are primarily crucial for magnetic protection. For instance, the magnetosphere of the Earth protects us from the radiation and wind emitted by the Sun and transported through its magnetic field. The only star-planet system that is fairly well known is the Sun-Earth system. This system is not unique and, hence, it is considered likely that other starexoplanet(s) systems should experience such magnetic interactions (Vidotto et al. 2013; Strugarek et al. 2014, 2015). In this context, constraining both stellar and planetary magnetic activity and magnetic field strength is thus essential when assessing planetary habitability. 


\subsection{Context}

During the last decade, stellar magnetic fields have been fairly well documented thanks to 3D topology reconstruction (see e.g. Donati et al. 2008; Petit et al. 2008; Morin et al. 2010; Mathur et al. 2015) and 3D dynamo simulations (Brun et al. 2004, 2015a; Browning 2008; Brown et al. 2010; Augustson et al. 2013, 2015; Palacios \& Brun 2014; Käpylä et al. 2014; Hotta et al. 2015; Guerrero et al. 2016).

More recently the interaction of stellar winds with planetary magnetospheres have just started to be intensively studied and modelled (e.g. Cohen et al. 2009; Vidotto et al. 2013; Strugarek et al. 2014, 2015; Vidotto et al. 2015). This interaction seems to have a very strong impact on the planetary habitability since a large percentage of the atmospheric component of a planet can be removed by these winds, which are thought to be strongly correlated to the X-ray luminosity known to be a good activity proxy (Wood et al. 2005; Vidotto et al. 2014, 2016).

As shown previously, the HZ location and width are not fixed but rather vary together with the stellar parameters along secular evolution. The question is then to know where the HZ is located regarding the stellar activity in order to estimate its impact on habitability.

\subsection{Rossby number and stellar magnetic field generation}

\subsubsection{Generalities}

The Rossby number quantifies the impact of rotation on the convective motion inside the star through a ratio of the inertial force to the Coriolis force. Thus, a Rossby number of less than one indicates that the Coriolis force is larger than the inertial force. In agreement with both theory and experiments (e.g. Durney \& Latour 1978; Noyes et al. 1984; Jouve et al. 2010; Calkins et al. 2015, 2016; Cheng \& Mahalov 2015; Brun et al. $2015 b$ ), a low Rossby number typically implies that the convective flows become quasi-2D and subsequently they generate a stronger and more spatio-temporally coherent kinetic helicity. The kinetic helicity is a critical component for a substantial $\alpha$ effect, which arises because of the action of small-scale vortices twisting the mean toroidal magnetic field into a mean poloidal magnetic field (e.g. Moffat 1978). Moreover, low Rossby number convection usually supports a strong solar-like differential rotation, which in turn gives rise to a strong $\Omega$ effect wherein the differential rotation winds up the mean poloidal magnetic field into a toroidal magnetic field. Hence, because both the $\alpha$ and $\Omega$ effects likely are more effective at a low Rossby number, one could expect more vigorous magnetic field generation within the convection zone and subsequent magnetic activity at the surface of the star.

\subsubsection{Prescriptions}

While stellar rotation has only a small effect on the location of the HZLs for the domain in stellar mass that we are considering (as shown in Fig. 4), it strongly impacts the stellar activity that is intimately linked to the Rossby regime in which the star is located (Brun et al. 2005). As defined before, the Rossby number evaluates the ratio of inertial to Coriolis force. It can be approximated by

$R o=\frac{P_{\mathrm{rot}}}{\tau_{\mathrm{conv}}}$,

where $P_{\text {rot }}$ is the surface rotation period of the star (in days), and $\tau_{\text {conv }}$ the convective turnover timescale (in days) at a given depth

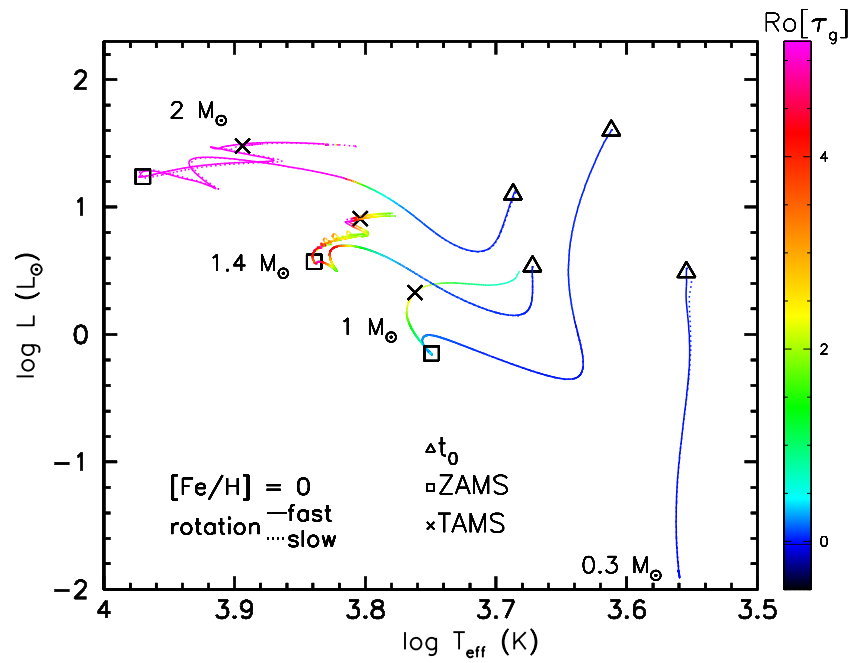

Fig. 10. Variation of the global Rossby number (colour-coded) along the evolution tracks in the HRD for the $0.3,1,1.4$, and $2 M_{\odot}, Z_{\odot}$ models for the fast- and slow-rotating cases (solid and dashed lines, respectively). The triangles, squares and crosses represent the location in the HRD of the age of the first time step $t_{0}$ in each STAREVOL model, ZAMS and TAMS, respectively.

within the stellar convective envelope (when it is present) given by

$\tau_{\text {conv }}(r)=\alpha H_{\mathrm{p}}(r) / V_{\mathrm{c}}(r)$,

where $\alpha, H_{\mathrm{p}}$, and $V_{\mathrm{c}}$ are the mixing-length parameters, local pressure scale height, and the local convective velocity. For a given star, the magnetic activity is expected to increase with decreasing Rossby number. Figure 10 shows the evolution in the HRD, for three selected stellar masses, of the global Rossby number estimated using rotating models with initial angular velocities as listed in Table 1 . The global convective turnover timescale associated with the global Rossby number $R o\left[\tau_{\mathrm{g}}\right]=$ $P_{\text {rot }} / \tau_{\mathrm{g}}$ is defined by

$\tau_{\mathrm{g}}=\int_{R_{\mathrm{b}}}^{R_{*}} \frac{\mathrm{d} r}{V_{\mathrm{c}}(r)}$

where $R_{\mathrm{b}}$ is the radius at the bottom of the convective envelope and $R_{*}$ the surface stellar radius.

\subsubsection{Rossby behaviour and correlation with the habitable zone}

In Fig. 10, we note three different kinds of behaviour: low-mass stars, solar-like stars, and intermediate-mass stars. The low-mass star $\left(0.3 M_{\odot}\right)$ stays in a very high stellar activity regime (low Rossby number) during its whole evolution due to a long convective turnover timescale because of a weak convective flow as the $V_{\mathrm{C}}$ scales as $\sqrt[3]{L_{*}}$. Therefore, solar and intermediate-mass stars experience a high activity regime on the PMS, which is followed by a lower activity phase (larger Ro numbers). There are two possible reasons for the increase of the Rossby number: either the rotation period $P_{\text {rot }}$ increases because of magnetic braking by stellar wind during the MS or the convective turnover time $\tau_{\text {conv }}$ decreases because of the reduction of the size of the convective envelope or the increase of the amplitude of the convective motions that scale as $\sqrt[3]{L_{*}}$. These two situations are represented in Fig. 10 by the solar-type and high-mass stars, respectively.

In the case of the solar-type stars, the evolution of the Rossby number is mainly controlled by the rotation period. The star maintains a high activity regime during the entire PMS phase 

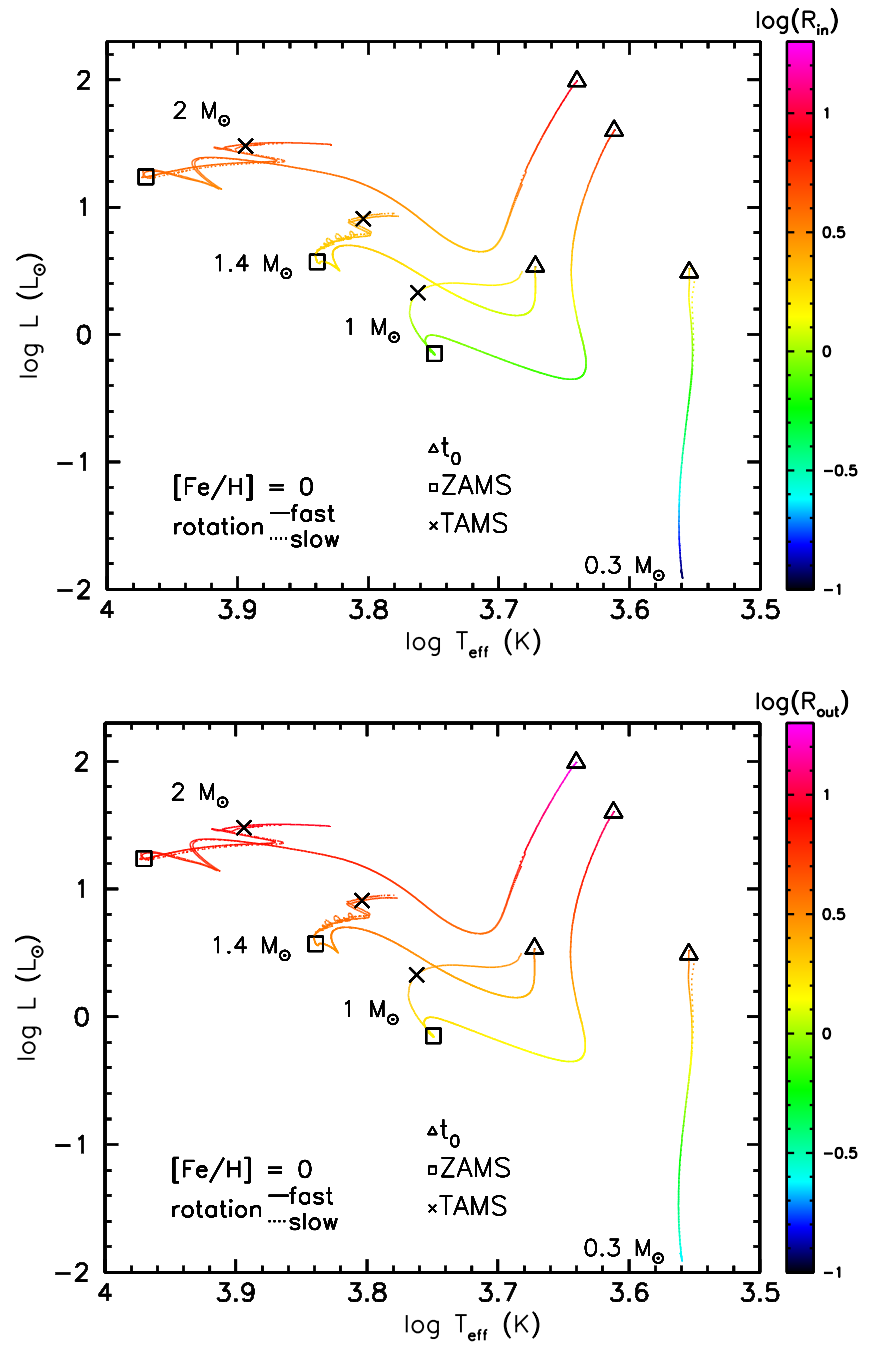

Fig. 11. Hertzsprung-Russell diagram of $0.3,1,1.4$, and $2 M_{\odot}$ stars with solar metallicity in fast (solid line) and slow (dashed line) rotation regime. The coloured gradient depicts the variation of $R_{\text {in }}$ and $R_{\text {out }}$ (top and bottom, respectively), expressed in $\mathrm{AU}$, along the stellar evolution. The triangles, squares, and crosses represent the location in the HRD of the age of the first time step $t_{0}$ in each STAREVOL models, ZAMS, and TAMS, respectively.

and it only starts to decrease after the ZAMS, i.e. when the rotation period starts to increase (smaller rotation rate) because of the magnetic braking (see Gallet \& Bouvier 2013, 2015, and references therein).

For the intermediate-mass star, the evolution of the Rossby number is controlled by the evolution of the internal structure. Figure 10 shows that the Rossby number already starts to increase during the mid-PMS phase (around $4 \times 10^{5} \mathrm{yr}$ ) because of radiative core development. The Rossby number thus rapidly increases above $R o=1$. When the star becomes almost radiative (size of the convective envelope lower than $1 \%$ of the size of the star, around $5 \mathrm{Myr}$ for a $2 M_{\odot}$ ) the Rossby number sharply rises towards high values and hence a likely low activity regime.

Figure 11 depicts the evolution of the inner and outer limits of the HZ, respectively, along the tracks in the HRD of a 0.3 , $1,1.4$, and $2 M_{\odot}$ stars with solar metallicity in fast and slow rotation regimes from the PMS to the end of the MS phase. The parameters $R_{\text {in }}$ and $R_{\text {out }}$ decrease during the Hayashi track (i.e. the HZ becomes closer to the star regardless of the stellar mass) while the global Rossby number is relatively small and around $R o \approx 10^{-3}-10^{-4}$ (see Fig. 10).
As the $1,1.4$, and $2 M_{\odot}$ stars evolve towards the ZAMS, their luminosity and effective temperature slowly increase which produces a small rise of both $R_{\text {in }}$ and $R_{\text {out }}$. Then, on the MS phase, the HZLs follow the intrinsic evolution of stellar luminosity and slightly increase all the way up to the end of the MS. Stars above $0.5-0.6 M_{\odot}$ reach a state of small HZLs during the midPMS phase.

For the $0.3 M_{\odot}$ star, this evolution is different since the stellar luminosity continuously decreases at almost constant effective temperature from the PMS to the end of the MS. The evolution of the stellar activity, that is simply estimated here thanks to the Rossby regime in which the star is lcoated, strongly depends on the stellar mass and, more specifically, on the internal structure of the considered star.

For the 1, 1.4, and $2 M_{\odot}$ stars, the stellar activity is high (i.e. Rossby number $<1$ ) along the PMS phase during which the HZLs are the closest to the star. For these stars, by opposition, the stellar activity is relatively low during the entire MS (and late PMS phase for the $2 M_{\odot}$ star) phase and may then not impact the long-term development of life during the earliest stages of the continuously habitable zone (hereafter CHZ; see Sect. 6). Conversely the $0.3 M_{\odot}$ star stays in a high activity regime during its whole life while the $\mathrm{HZ}$ continuously moves closer to the star.

While there is no definite correlation between the activity regime of the star and the location of the HZLs, the stellar activity must be taken into account and, more specifically, for M dwarfs, to assess the habitability of a planet since currently almost all detected potentially habitable planets orbit low-mass stars (i.e. around $0.3 M_{\odot}$ ). For this stellar mass regime, and as we showed above, the stellar activity cannot be neglected and may have a strong impact on life development and complexification.

Moreover, short-term extreme events, such as Carringtonlike and superflares events (Maehara et al. 2012), can occur. Thus using only the Rossby number to assess space weather condition is a zero order approach that needs to be further refined in future studies.

\subsection{Magnetic flux and magnetospheric protection}

In addition to be inside the HZ, a planet also needs to possess an effective magnetospheric protection to be considered habitable. There are few questions that are worth answering: what is the maximum stellar magnetic field (thus magnetic flux) that a planet found inside the HZ can handle given its own magnetic field? What is the minimum planetary magnetic field required to handle a sun-like star magnetic field evolution?

General theoretical robust scaling laws for the amplitude of a magnetic field as a function of parameters, computed only owing to stellar evolution codes, are not yet available in the literature. Either the magnetic field amplitude is computed from complex 3D numerical simulations and simplified asymptotic balances (e.g. Brun et al. 2015a; Augustson et al. 2015) or from a simplified relation by assuming the magnetic field at the thermal equilibrium with the stellar photosphere (e.g. Johns-Krull \& Valenti 2000; Cranmer \& Saar 2011). Therefore, we rather directly use the observed trends of the magnetic field strength as a function of time, rotation, and Rossby number.

Folsom et al. (2016) provide a compilation of magnetic field measurements from three large spectropolarimetric surveys: HMS (the History of the Magnetic Sun in the framework of the Toupies ${ }^{4}$ project), BCool, and MaPP ${ }^{5}$. Their Fig. 9 shows clear

\footnotetext{
4 Toupies: TOwards Understanding the sPin Evolution of Stars. 5 MaPP: MAgnetic Protostars and Planets.
} 
trends of the mean large-scale magnetic field strength with age, Rossby, and also rotation (but only for high rotation period).

In the framework of an exoplanet orbiting a star with a large enough orbital radius (larger than few tenth of stellar radius) the magnetic field to take into account is the large-scale magnetic field. Folsom et al. (2016) found that

$B_{*}=1680.9 \times t^{-0.74308} \mathrm{G}$,

where $t$ is the age expressed in Myr, and

$B_{*} \propto R o^{-1}$.

We normalised this latter expression so as to reproduce the Sun's magnetic field strength,

$B_{*}=B_{\odot} \frac{R o_{\odot}}{R o_{*}}$,

with $B_{\odot}=2$ gauss, $R o_{\odot}=1.96$, while $R o_{*}$ is estimated using $\tau_{\mathrm{g}}$.

Vidotto et al. (2013) provided a direct relation between the minimum planetary magnetic field, required for an effective magnetic protection, and the stellar magnetic field,

$B_{\mathrm{p}}^{\min } \simeq 16052 P_{\mathrm{B}, *}\left(R_{\mathrm{orb}}\right)^{1 / 2}$,

where

$P_{\mathrm{B}, *}\left(R_{\mathrm{orb}}\right)=\frac{B_{\mathrm{SS}}^{2}}{8 \pi}\left(\frac{R_{\mathrm{SS}}}{R_{\mathrm{orb}}}\right)^{4}$,

is the magnetic pressure with $R_{\mathrm{SS}}$ the source surface radius (assumed to be $2.5 R_{*}$, which is known not to be valid for all ages and rotation rates; see Réville et al. 2015), $B_{\mathrm{SS}}$ the magnetic field at $R_{\mathrm{SS}}, R_{\mathrm{orb}}$ the orbital radius of the exoplanet, and $B_{\mathrm{p}}^{\mathrm{min}}$ the minimum planetary magnetic field required for an effective magnetic protection. Here we assume a simple region of interaction and we neglect the planetary magnetic field orientation (Saur et al. 2013; Strugarek et al. 2015) as well as the activity cycle and grand maxima phase.

We considered an Earth mass planet situated at 1 AU. By coupling Eqs. (13) and (14), the minimum planetary magnetic field can be expressed as a function of stellar and planetary parameters as follows:

$B_{\mathrm{p}}^{\min } \simeq 16052\left[\frac{B_{*}^{2}}{8 \pi}\left(\frac{2.5 R_{*}}{1 \mathrm{AU}}\right)^{4}\right]^{1 / 2}$.

We note that $B_{\mathrm{p}}^{\min }$ is proportional to the squared stellar radius.

At the age of the Sun, an Earth-like planet needs a magnetic field of at least $0.8 \mathrm{G}$ to be protected from a solar-like magnetic field of $1.88 \mathrm{G}$ (see Fig. 12), which is consistent with the measured values of the Earth $\left(B_{\oplus} \approx 0.5 \mathrm{G}\right)$ and Sun $\left(B_{\odot} \approx 2 \mathrm{G}\right)$ magnetism. These small differences between numerical and observed magnetic fields are due to the observed magnetic prescription employed, which only provides an order of magnitude estimate of the magnetic field. Because more massive stars are less active on the MS (see Fig. 10), $B_{\mathrm{p}}^{\min }$ also globally decreases towards higher masses. However, since $B_{\mathrm{p}}^{\min }$ is proportional to the squared stellar radius, the minimum planetary magnetic field increases during the MS as the stellar radius increases. While the evolution of $B_{\mathrm{p}}^{\min }$ in the case of the $1 M_{\odot}$ star is dominated by the evolution of the stellar magnetic field controlled by the rotation period of the star through the Rossby number, in the case of the $2 M_{\odot}$ star this evolution is controlled by the evolution of the stellar radius that slightly increases during the MS phase while the magnetic field remains more or less constant.

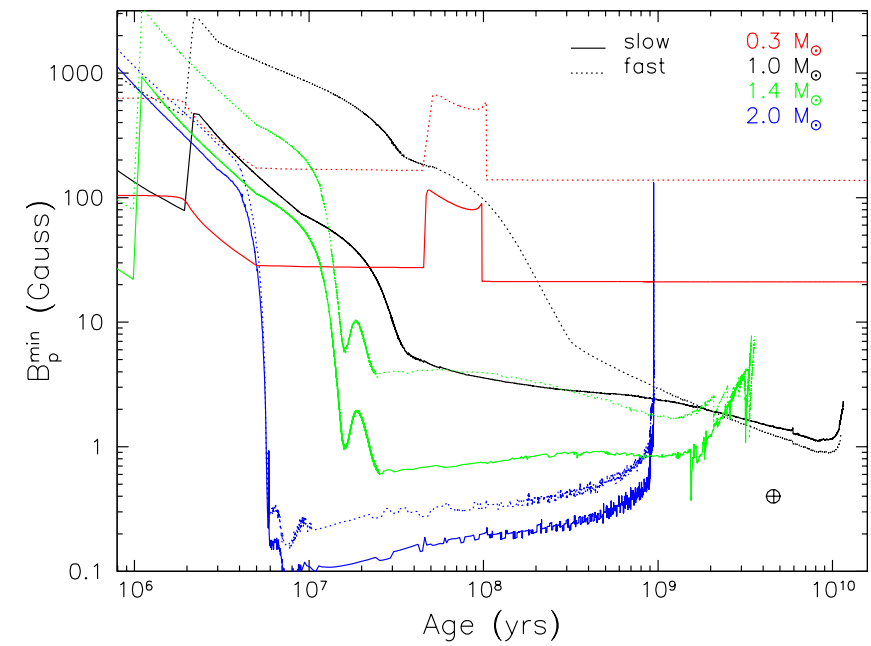

Fig. 12. Minimum planetary magnetic field required by a $1 M_{\oplus}$ orbiting a $0.3,1,1.4$, and $2 M_{\odot}$ at $1 \mathrm{AU}$ to produce an effective magnetic protection. Solid and dashed lines represent slow and fast rotators, respectively. The Earth is represented by the $\oplus$ symbol.

The minimum planetary magnetic field for the $1 M_{\odot}$ first starts to decrease up to $2 \mathrm{Myr}$ when the radiative core appears inside the star. This stable core development decreases the convective turnover timescale and thus decreases the Rossby number of the star, which leads to the increase of the stellar magnetic field strength and $B_{\mathrm{p}}^{\min }$. This diminution then continues up to the ZAMS, where the size of the core remains almost constant. After the ZAMS the decrease of $B_{*}$ (and $B_{\mathrm{p}}^{\min }$ ) is entirely controlled by the rotation, as $R_{*}$ is almost fixed, which continuously decreases because of the stellar magnetic wind braking. Beyond the MS turn-off and during the RGB phase both magnetic fields slightly increase as the radius of the star increases so as to maintain a surface hydrostatic equilibrium.

The evolution of $B_{\mathrm{p}}^{\mathrm{min}}$ for the $2 M_{\odot}$ follows more or less the same trends except that the internal structure of this star is fixed at the beginning of its evolution. The parameter $B_{\mathrm{p}}^{\min }$ first starts to decrease because of the stellar contraction, then reaches a plateau at ZAMS that lasts until the red giant phase where $B_{\mathrm{p}}^{\min }$ sharply increases.

In the case of the $0.3 M_{\odot}$ star, a radiative core starts to develop just before $100 \mathrm{Myr}$ and lasts for only $50 \mathrm{Myr}$. The growth of the core sharply decreases the convective turnover time (as the size of the convective envelope decreased) producing the rise of the stellar and thus planetary magnetic field in our simplified approach. As a consequence, the minimum planetary magnetic field rises up to $100 \mathrm{G}$ during this brief phase. As a $0.3 M_{\odot}$ is fully convective during almost its entire life and as the magnetic braking is not efficient during the MS, the minimum planetary magnetic field remains constant throughout the entire evolution of such a low-mass star.

During the MS, the minimum magnetic field $B_{\mathrm{p}}^{\min }$ in the case of a solar-type star is about one order of magnitude lower than $B_{\mathrm{p}}^{\mathrm{min}}$ for an M-dwarf star.

Inversely, if we fix the planetary magnetic field we can study the evolution of the maximum stellar magnetic field that a planet can handle,

$B_{*}^{\max } \simeq \frac{B_{\mathrm{p}}^{\min }}{16052}\left[8 \pi\left(\frac{1 \mathrm{AU}}{2.5 R_{*}}\right)^{4}\right]^{1 / 2}$.

Figure 13 shows the temporal evolution of $B_{*}^{\max }$ as a function of the stellar mass for two planetary magnetic field strengths $(0.5$ 
F. Gallet et al.: Impacts of stellar evolution and dynamics on the habitable zone: The role of rotation and magnetic activity

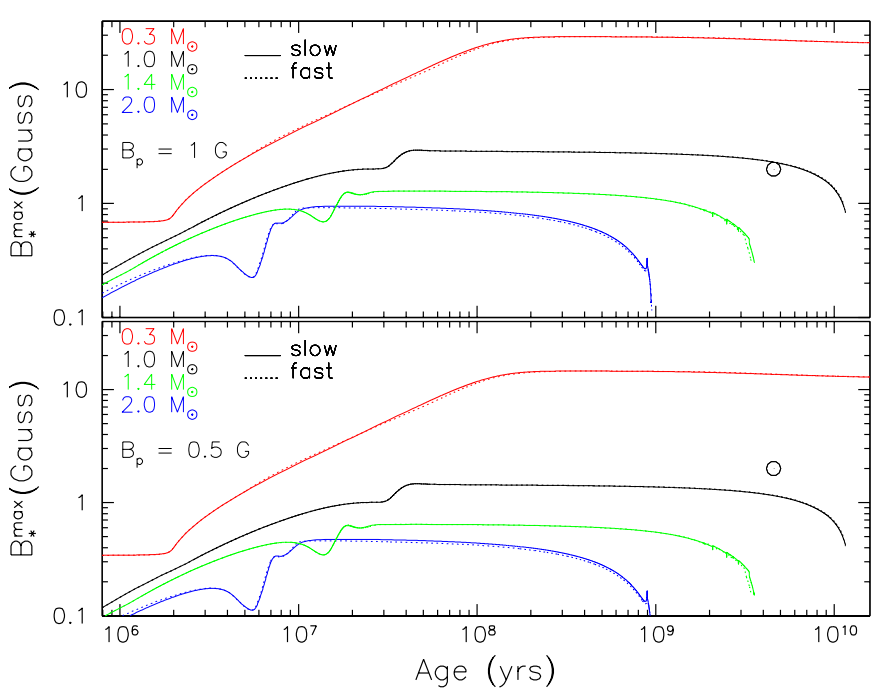

Fig. 13. Maximum stellar magnetic field that a $1 M_{\oplus}$ orbiting a $0.3,1$, 1.4 , and $2 M_{\odot}$ star at $1 \mathrm{AU}$ can sustain given its fixed magnetic field. Solid and dashed lines represent slow and fast rotators, respectively. The Sun is represented by the $\odot$ symbol.

and 1 gauss). As the star evolves, its radius first decreases, which is translated, at fixed planetary magnetic field, by an increase of the maximum stellar magnetic field that the planet can support (since $B_{*}^{\max }$ evolves as $R_{*}^{-2}$, see Eq. (16)). During the MS this maximum magnetic field reaches a plateau as the stellar internal structure remains almost constant. During the RGB, and following the stellar expansion, $B_{*}^{\max }$ slowly decreases. As expected a more magnetized planet can handle a more intense stellar magnetic field strength. Equation (16) only takes into account the structural aspect (through the evolution of $R_{*}$ ) and no dynamical evolution. Moreover, the effect of the orientation of the planetary magnetic field with respect to that of the star is here also not taken into account.

\section{Conclusions}

To assess the habitability of a class I exoplanet (i.e. with surface liquid water), the HZLs first needs to be precisely defined. In the literature the $\mathrm{HZ}$ is considered at a specific time of the evolution (i.e. considering the properties of the present-day Sun). However, we show in this paper that the variations of the HZLs are important as stars evolve and that the HZLs depend on stellar mass and metallicity because they strongly affect the stellar luminosity and effective temperature. These two quantities are marginally affected by a change in rotation rate. However, stellar rotation has a major role on tidal and magnetic torques evolution (Mathis 2015; Strugarek et al. 2015; Bolmont \& Mathis 2016) that then dramatically affect the orbital evolution of a given planet. We have shown in this work that a correlation exists between the stellar activity and location of the $\mathrm{HZ}$ along the stellar evolution, indeed the HZLs are the closest to the star when it is in a high stellar active regime. Even though the impact of stellar activity on the HZLs is not straightforward to assess, it may impact the atmospheric conditions of the planet, which in turn impacts the location of the HZLs. In this work we showed that M-dwarf stars possess a strong stellar activity during their entire life, while more massive stars (solar-type and intermediatemass stars) only exhibit strong activity during their early evolution (i.e. during their PMS phase). As the stellar mass decreases its magnetic field strength globally increases, at least during the MS phase during which a star-planet system passes almost its entire evolution. As a consequence, during the MS the minimum magnetic field required by an exoplanet for an efficient magnetospheric protection increases for decreasing mass. This could pose a problem in finding habitable planets as exoplanets inside the habitable zone are currently almost only found around M-dwarf stars.

With this work we provide better constraints on the HZLs by using the stellar evolution code STAREVOL, which incorporates current mechanisms of stellar evolution physics. The final purpose of this study is to provide the community with an on-line tool dedicated to the study of the evolution of the habitability (link to the HZ and planet's orbital motion) of a potential exoplanet. This tool will then be very useful for example for the CHEOPS (Broeg et al. 2013), TESS (Ricker et al. 2015), PLATO (Rauer et al. 2014), and SPIRou (Moutou et al. 2015) community. Thanks to the models presented in this paper we can predict the HZLs for numerous star-planet configurations (and for three planetary masses), and can already provide the community with a dedicated grid of stellar models to estimate this quantity.

Additionally, the evolution of the planet's orbital motion is expected to strongly impact the habitability by moving the planet inside or outside the HZ. Detailed analysis of tidal dissipation in both the planet and star is then crucial to study this evolution (e.g. Mathis \& Remus 2013; Ogilvie 2014). This mechanism will be soon implemented in the STAREVOL code using the formalism of Ogilvie (2013) and Mathis (2015), and the corresponding results will be presented in a forthcoming paper.

Acknowledgements. We thank the referee for her/his comments that allowed us to improve the article. The authors are grateful to K. Augustson for valuable discussions and inputs on stellar activity. This work results within the collaboration of the COST Action TD 1308 and by the grant ANR 2011 Blanc SIMI5-6 02001 "Toupies: Towards understanding the spin evolution of stars" (http://ipag.osug.fr/Anr_Toupies/). The authors acknowledge financial support from the Swiss National Science Foundation (FNS) and from the French Programme National de Physique Stellaire PNPS of CNRS/INSU. A.S.B. acknowledges support by CNES through Solar Orbiter and Plato fundings as well as ERC STARS2 207430, Solar Predict 640997 and FP7 Spaceinn grants. S. Mathis acknowledges funding by the European Research Council through ERC SPIRE grant 647383

\section{References}

Amard, L., Palacios, A., Charbonnel, C., Gallet, F., \& Bouvier, J. 2016, A\&A, 587, A 105

Asplund, M., Grevesse, N., \& Sauval, A. J. 2005, in Cosmic Abundances as Records of Stellar Evolution and Nucleosynthesis, eds. T. G. Barnes, III, \& F. N. Bash, ASP Conf. Ser., 336, 25

Asplund, M., Grevesse, N., Sauval, A. J., \& Scott, P. 2009, ARA\&A, 47, 481

Augustson, K. C., Brun, A. S., \& Toomre, J. 2013, ApJ, 777, 153

Augustson, K. C., Brun, A. S., Miesch, M., \& Toomre, J. 2015, in 18th Cambridge Workshop on Cool Stars, Stellar Systems, and the Sun, eds. G. T. van Belle, \& H. C. Harris, 451

Batalha, N. M., Borucki, W. J., Bryson, S. T., et al. 2011, ApJ, 729, 27

Bell, C. P. M., Naylor, T., Mayne, N. J., Jeffries, R. D., \& Littlefair, S. P. 2013, MNRAS, 434, 806

Bolmont, E., \& Mathis, S. 2016, Celest. Mech. Dyn. Astron., 126, 275

Borucki, W. J., Koch, D. G., Basri, G., et al. 2011, ApJ, 736, 19

Broeg, C., Fortier, A., Ehrenreich, D., et al. 2013, EPJ Web Conf., 47, 03005

Brown, B. P., Browning, M. K., Brun, A. S., Miesch, M. S., \& Toomre, J. 2010, ApJ, 711, 424

Browning, M. K. 2008, ApJ, 676, 1262

Brun, A. S., Miesch, M. S., \& Toomre, J. 2004, ApJ, 614, 1073

Brun, A. S., Browning, M. K., \& Toomre, J. 2005, ApJ, 629, 461

Brun, A. S., Browning, M. K., Dikpati, M., Hotta, H., \& Strugarek, A. 2015a, Space Sci. Rev., 196, 101

Brun, A. S., García, R. A., Houdek, G., Nandy, D., \& Pinsonneault, M. 2015b, Space Sci. Rev., 196, 303

Calkins, M. A., Julien, K., \& Marti, P. 2015, Proc. R. Soc. Lond. Ser. A, 471, 20140689 
Calkins, M. A., Julien, K., Tobias, S. M., Aurnou, J. M., \& Marti, P. 2016, Phys. Rev. E, 93, 023115

Cheng, B., \& Mahalov, A. 2015, ArXiv e-prints [arXiv: 1508.00633]

Cohen, O., Drake, J. J., Kashyap, V. L., et al. 2009, ApJ, 704, L85

Cranmer, S. R., \& Saar, S. H. 2011, ApJ, 741, 54

Decressin, T., Mathis, S., Palacios, A., et al. 2009, A\&A, 495, 271

Degl'Innocenti, S., Prada Moroni, P. G., Marconi, M., \& Ruoppo, A. 2008, Ap\&SS, 316, 25

Donati, J.-F., Morin, J., Petit, P., et al. 2008, MNRAS, 390, 545

Dumusque, X., Pepe, F., Lovis, C., et al. 2012, Nature, 491, 207

Durney, B. R., \& Latour, J. 1978, Geophys. Astrophys. Fluid Dyn., 9, 241

Endal, A. S., \& Sofia, S. 1976, ApJ, 210, 184

Ferreira, J. 2013, in EAS Pub. Ser. 62, eds. P. Hennebelle, \& C. Charbonnel, 169

Folsom, C. P., Petit, P., Bouvier, J., et al. 2016, MNRAS, 457, 580

Forget, F. 2013, Int. J. Astrobiol., 12, 177

Gallet, F., \& Bouvier, J. 2013, A\&A, 556, A36

Gallet, F., \& Bouvier, J. 2015, A\&A, 577, A98

Gallet, F., Charbonnel, C., \& Amard, L. 2016, Origins of Life and Evolution of the Biosphere, 46, 395

Güdel, M., Dvorak, R., Erkaev, N., et al. 2014, Protostars and Planets VI, 883

Guerrero, G., Smolarkiewicz, P. K., de Gouveia Dal Pino, E. M., Kosovichev,

A. G., \& Mansour, N. N. 2016, ApJ, 819, 104

Hart, M. H. 1976, BAAS, 8, 540

Hayashi, C. 1961, PASJ, 13, 450

Heller, R., Leconte, J., \& Barnes, R. 2011, A\&A, 528, A27

Henyey, L. G., Lelevier, R., \& Levée, R. D. 1955, PASP, 67, 154

Holman, M. J., Fabrycky, D. C., Ragozzine, D., et al. 2010, Science, 330, 51

Hotta, H., Rempel, M., \& Yokoyama, T. 2015, ApJ, 803, 42

Johns-Krull, C. M., \& Valenti, J. A. 2000, in Stellar Clusters and Associations

Convection, Rotation, and Dynamos, eds. R. Pallavicini, G. Micela, \& S. Sciortino, ASP Conf. Ser., 198, 371

Johnstone, C. P., Güdel, M., Brott, I., \& Lüftinger, T. 2015, A\&A, 577, A28

Jouve, L., Brown, B. P., \& Brun, A. S. 2010, A\&A, 509, A32

Käpylä, P. J., Käpylä, M. J., \& Brandenburg, A. 2014, A\&A, 570, A43

Kasting, J. F. 2010, in Pathways Towards Habitable Planets, eds. V. Coudé du Foresto, D. M. Gelino, \& I. Ribas, ASP Conf. Ser., 430, 3

Kasting, J. F., Whitmire, D. P., \& Reynolds, R. T. 1993, Icarus, 101, 108

Kopparapu, R. K., Ramirez, R., Kasting, J. F., et al. 2013, ApJ, 765, 131

Kopparapu, R. K., Ramirez, R. M., SchottelKotte, J., et al. 2014, ApJ, 787, L29

Lammer, H., Bredehöft, J. H., Coustenis, A., et al. 2009, A\&ARv, 17, 181

Leconte, J., Forget, F., Charnay, B., Wordsworth, R., \& Pottier, A. 2013a, Nature, 504,268

Leconte, J., Forget, F., Charnay, B., et al. 2013b, A\&A, 554, A69

Leconte, J., Wu, H., Menou, K., \& Murray, N. 2015, Science, 347, 632

Linsenmeier, M., Pascale, S., \& Lucarini, V. 2015, Planet. Space Sci., 105, 43

Lissauer, J. J., Fabrycky, D. C., Ford, E. B., et al. 2011, Nature, 470, 53

Lodders, K. 2010, Astrophys. Space Sci. Proc., 16, 379

Maeder, A. 2009, Physics, Formation and Evolution of Rotating Stars (Berlin, Heidelberg: Springer)

Maeder, A., \& Zahn, J.-P. 1998, A\&A, 334, 1000

Maehara, H., Shibayama, T., Notsu, S., et al. 2012, Nature, 485, 478

Mathis, S. 2015, A\&A, 580, L3

Mathis, S., \& Remus, F. 2013, in Lect. Notes Phys. 857, eds. J.-P. Rozelot, \& C. Neiner (Berlin: Springer Verlag), 111
Mathis, S., \& Zahn, J.-P. 2004, A\&A, 425, 229

Mathis, S., Auclair-Desrotour, P., Guenel, M., Gallet, F., \& Le Poncin-Lafitte, C. 2016, A\&A, 592, A33

Mathur, S., Augustson, K. C., Brun, A. S., Garcia, R. A., \& Metcalfe, T. S. 2015, in 18th Cambridge Workshop on Cool Stars, Stellar Systems, and the Sun, eds. G. T. van Belle, \& H. C. Harris, 365

Matt, S. P., Brun, A. S., Baraffe, I., Bouvier, J., \& Chabrier, G. 2015, ApJ, 799, L23

Mayor, M., \& Queloz, D. 1995, Nature, 378, 355

Mayor, M., Bonfils, X., Forveille, T., et al. 2009, A\&A, 507, 487

Moffat, J. W. 1978, Phys. Rev. D, 17, 1965

Morin, J., Donati, J.-F., Petit, P., et al. 2010, MNRAS, 407, 2269

Moutou, C., Boisse, I., Hébrard, G., et al. 2015, in SF2A-2015: Proc. of the Annual meeting of the French Society of Astronomy and Astrophysics, eds. F. Martins, S. Boissier, V. Buat, L. Cambrésy, \& P. Petit, 205

Noyes, R. W., Weiss, N. O., \& Vaughan, A. H. 1984, ApJ, 287, 769

Ogilvie, G. I. 2013, MNRAS, 429, 613

Ogilvie, G. I. 2014, ARA\&A, 52, 171

Palacios, A., \& Brun, A. S. 2014, in IAU Symp. 302, eds. P. Petit, M. Jardine, \& H. C. Spruit, 363

Palacios, A., Talon, S., Charbonnel, C., \& Forestini, M. 2003, A\&A, 399, 603

Palacios, A., Charbonnel, C., Talon, S., \& Siess, L. 2006, A\&A, 453, 261

Petit, P., Dintrans, B., Solanki, S. K., et al. 2008, MNRAS, 388, 80

Quintana, E. V., Barclay, T., Raymond, S. N., et al. 2014, Science, 344, 277

Rauer, H., Catala, C., Aerts, C., et al. 2014, Exp. Astron., 38, 249

Reiners, A., \& Mohanty, S. 2012, ApJ, 746, 43

Réville, V., Brun, A. S., Strugarek, A., et al. 2015, ApJ, 814, 99

Ricker, G. R., Winn, J. N., Vanderspek, R., et al. 2015, J. Astron. Telescopes, Instruments, and Systems, 1, 014003

Saur, J., Grambusch, T., Duling, S., Neubauer, F. M., \& Simon, S. 2013, A\&A, 552, A119

Selsis, F., Kasting, J. F., Levrard, B., et al. 2007, A\&A, 476, 1373

Stevenson, D. J. 2003, Earth Plan. Sci. Lett., 208, 1

Strugarek, A., Brun, A. S., Matt, S. P., \& Réville, V. 2014, ApJ, 795, 86

Strugarek, A., Brun, A. S., Matt, S. P., \& Réville, V. 2015, ApJ, 815, 111

Tognelli, E., Prada Moroni, P. G., \& Degl'Innocenti, S. 2011, A\&A, 533, A109

Torres, G., Kipping, D. M., Fressin, F., et al. 2015, ApJ, 800, 99

Truitt, A., Young, P. A., Spacek, A., Probst, L., \& Dietrich, J. 2015, ApJ, 804, 145

Udry, S., Bonfils, X., Delfosse, X., et al. 2007, A\&A, 469, L43

Underwood, D. R., Jones, B. W., \& Sleep, P. N. 2003, Int. J. Astrobiol., 2, 289

Valle, G., Dell'Omodarme, M., Prada Moroni, P. G., \& Degl'Innocenti, S. 2014, A\&A, 567, A133

Vidotto, A. A., Jardine, M., Morin, J., et al. 2013, A\&A, 557, A67

Vidotto, A. A., Gregory, S. G., Jardine, M., et al. 2014, MNRAS, 441, 2361

Vidotto, A. A., Fares, R., Jardine, M., Moutou, C., \& Donati, J.-F. 2015, MNRAS, 449, 4117

Vidotto, A. A., Donati, J.-F., Jardine, M., et al. 2016, MNRAS, 455, L52

Wood, B. E., Müller, H.-R., Zank, G. P., Linsky, J. L., \& Redfield, S. 2005, ApJ, $628, \mathrm{~L} 143$

Young, P. A., \& Arnett, D. 2005, ApJ, 618, 908

Zahn, J.-P. 1992, A\&A, 265, 115

Zahn, J.-P., \& Bouchet, L. 1989, A\&A, 223, 112 